\title{
Three-dimensional streaming flows driven by oscillatory boundary layers
}

\author{
José A. Nicolás, José M. Vega \\ ETSI Aeroniaticos, Lnirersidad Politécnica de Madrid, Plaza Cardenal Cisneros, 3, 28040) Madrid, Spain
}

\begin{abstract}
Three-dimensional (3D) oscillatory boundary layers attached to deformable solid walls and free boundaries of general form are analyzed via matched asymptotic expansions, to obtain the time-averaged tangential velocities and tangential stresses, respectively, at the edge of the layers. These provide the appropriate boundary conditions that are to be used to calculate the streaming flow in the bulk, outside the boundary layers. The resulting formulae generalize to 3D the well-known expressions due to Schlichting (Phys. Z. 33 (1932) 327) and Longuet-Higgins (Philos. Trans. R. Soc. A 245 (1953) 535 ).

(c) 2003 Published by The Japan Society of Fluid Mechanics and Elsevier Science B.V. All rights reserved.
\end{abstract}

Kẹwords: Streaming llows; Viscous mean flows; Oscillatory boundary layers

\section{Introduction}

Nearly inviscid oscillatory flows involve oscillatory boundary layers near solid walls and free boundaries. Reynolds stresses exhibit a non-zero temporal mean in these boundary layers, where they drive a meam flow, as first shown in a pioneering work by Lord Rayleigh (1883), in his explanation of the anomalous sand accumulation at the bottom of a vertically vibrated container and of dust at the walls of sound tubes, observed 50 years earlier by Faraday (1831). Rayleigh made a careful analysis of the (nowadays called) boundary layer attached to a no slip boundary and obtained the steady mean flow responsible for sand or dust nonuniform accumulation near the boundary, but did not pay much attention to the fact that the horizontal mean flow velocity is non-zero at the internal edge of the boundary layer, and thus is able to drive a mean flow in the bulk. This was done 50 years 
later by Schlichting (1932) (see also Schlichting (1968)) and further pursued by Longuct-Higgins (1953), who also calculated the streaming flow within the boundary layer attached to a free boundary, and the associated time-averaged shear stress that again was non-zero at the internal edge of the layer. An important well known (Batchelor, 1967) property is that the time-averaged velocity or stress converges to a generally non-zero value as viscosity goes to zero, while it would vanish if viscosity is zero (when the boundary laver is absent), which is not surprising due to the singular perturbation character of the inviscid limit. More recently, the mean flow induced by no slip boundaries (also called steady streaming, or acoustic streaming Rilcy, 2001) has been studicd in conncetion with flows in blood vessels (Padmanabhan and Pedley, 1987), generation of mean motions in the ear (Lighthill, 1992), interaction of sound waves with bodies (Riley, 1992), and flows around vibrating bodies (Yan et al., 1993). And the streaming flow produced in the boundary layer attached to a vibrating free boundary is of interest in water wave theory (Phillips, 1977; Liu and Davis, 1977; Craik, 1982, 1985; Iskandarani and Liu, 1991 and references therein) and has been shown to play a role in the instability of the ocean to Langmuir circulations (Leibovich, 1983). These flows have also been studied in connection with capillary waves (Mollot et al., 1993) and in conjunction with thermal effects (Nicolás and Vega, 1996: Nicolás et al., 1997, 1998; Lyubimov et al., 1997), intending to control thermocapillary convection (Anilkumar et al., 1993), which is undesirable in materials processing in microgravity (Kuhlmann, 1999). Most of these works dealt with the two-dimensional (2D) case and used the 2D formulae derived by Schlichting (1968) and Longuet-Higgins (1953) for the boundary conditions at the edge of the boundary layers when solving the mean flow equations in the bulk. When dealing with 3D problems instead, the boundary conditions must be derived in each case due to, in words of Lighthill (1992, p. 576), "a regrettable absence of information about streaming generated within three-dimensional Stokes boundary layers". The main object of this paper is precisely to fill this gap, deriving general formulae for the 3D case. These are not straightforward extensions of (and cannot be guessed from) their 2D counterparts; compare, e.g. (4.6) and (4.8), or (5.26) and (5.32) below. And some care must be taken in extending 2D results to axisymmetric problems, see the example at the end of Section 5.3. Seeking for reasonable generality, we shall consider oscillatory boundary layers attached to both deformable solids and free boundaries that are oscillating around a surface of arbitrary smooth shape. Still, in all works mentioned above, the streaming flow was a by-product of the primary oscillatory flow, which happens when the forcing frequency is not a natural frequency of the system. If instead the oscillations are resonant, the mean flow does aflect the weakly-non-linear dynamics of the surface waves themselves at leading order (producing a term in the amplitude equations that is of the same order as the cubic non-linearity that is usually retained), as it has been recently shown (Vega et al., 2001; Higuera et al., 2001; Martin et al., 2002; Knobloch and Vega, 2002; Knobloch et al., 2002). This is consistent with the fact that steady circulations are well known to affect surface wave dynamics (Milewsky and Benney, 1995; Mashayek and Ashgriz, 1998).

Against this background, the remaining of the paper is organized as follows. In order to illustrate the role of the oscillatory boundary layers in the forcing of the streaming flow in the bulk, and to explain the difficulties to be encountered in 3D, we consider in Section 2 a fairly simple 2D problem, namely a vibrating containcr filled with liquid. The 3D problem is formulated in Scetion 3, and the boundary layers attached to 3D solid walls and free boundaries are analyzed in Sections 4 and 5, respectively. For completeness we also briefly consider in Section 6 a boundary layer attached to a deformable solid, which is of interest in, e.g. Biophysics (Padmanabhan and Pedley, 1987; Lighthill, 1992). Finally, some concluding remarks are made in Section 7. 


\section{A simple 2D problem}

We consider a horizontal 2D rectangular container that is vibrating horizontally and harmonically with an amplitude $a$ and a frequency $\omega$ (Fig, 1). We use the unperturbed height of the liquid $\ell$ and $a^{-1}$ as characteristic length and time for non-dimensionalization (the velocity being non-dimensionalized with $(\omega)$ ), to write the governing equations and boundary conditions in a vibrating, rectangular coordinate system as

$$
\begin{aligned}
& u_{x}+v_{y}=0, \\
& u_{t}+v\left(u_{y}-v_{x}\right)=-p_{x}+R^{-1}\left(u_{x x}+u_{y y}\right)+\varepsilon \cos l, \\
& v_{t}-u\left(u_{y}-v_{x}\right)=-p_{y}+R^{-1}\left(v_{x x}+v_{y y}\right), \\
& u=v=0 \quad \text { at } y=-1 \quad \text { and at } x= \pm 1, \\
& v=f_{t}+u f_{x}, \quad\left(u_{y}+v_{x}\right)\left(1-f_{x}^{2}\right)+2\left(v_{y}-u_{x}\right) f_{x}=0, \\
& p-\left(u^{2}+v^{2}\right) / 2-G f+\frac{T f_{x x}}{\left(1+f_{x}^{2}\right)^{3 / 2}}=\frac{2 R^{-1}\left[v_{y}+u_{x} f_{x}^{2}-\left(u_{y}+v_{x}\right) f_{x}\right]}{1+f_{x}^{2}} \text { at } y=f, \\
& f=0 \quad \text { at } x= \pm 1 .
\end{aligned}
$$

Here $u$ and $v$ are the horizontal and vertical velocity components, $p\left(=\right.$ pressure $+G y+\left(u^{2}+v^{2}\right) / 2$, where $\rho$ is the density and $g$ is the gravitational acceleration) is the hydrostatic, stagnation pressure, $f$ is the free boundary elevation (measured from the undisturbed position), and $\left.G=g /(t, a)^{2}\right)$ and $T=\sigma /\left(\rho \ell^{3} \alpha^{2}\right)$ are non-dimensional measures of gravity and surface tension, respectively, where $\sigma$ is the surface tension coeflicient. For simplicity we assume that $G \sim T \sim 1$, that the aspect ratio of

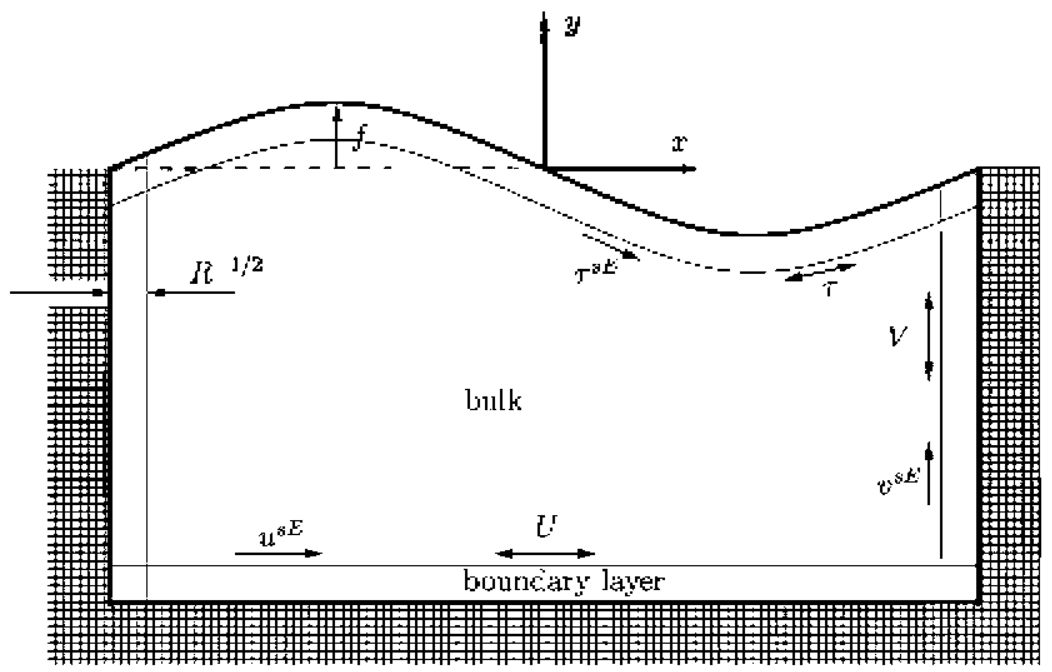

Fig. 1. 
the container is 2, and that the liquid fills the container up to the borders of the lateral walls, see below. The following basic assumptions concern the non-dimensional vibrating amplitude and the Reynolds-Strouhal number (also known as pulsatile Reynolds number Sobey, 1980),

$$
\because=a / t \ll 1, \quad R=w t^{2} / v \equiv\left(f / t_{\mathrm{b}}\right)^{2} \gg 1,
$$

where $v$ is the kinematic viscosity and $\ell_{\mathrm{bl}}=\sqrt{v / \omega}$ is the thickness of the oscillatory boundary layers. With this notation, $R=R e \cdot S t$, where $R e=a t b / v$ is the Reynolds number and $\mathrm{St}=t / a \equiv a^{-1}$ is the Strouhal number. The first assumption (2.7) implies that convection is small compared to the non-steady part of inertia and because of the second assumption (2.7), two oscillatory boundary layers develop near the bottom and the free surface. Thus convective terms will be neglected at leading order, the boundary layer equations will be linear, and boundary-layer separation (Smith, 1986) and other non-linear phenomena (Sobcy, 1980; Stephanoff et al, 1980; Pedley, 2000 and references given therein) that appears at finite Strouhal number will be absent in the analysis below.

In this limit, we seck the following expansions outside boundary layers (in the bulk)

$$
\begin{aligned}
(u, v, p, f)= & \varepsilon\left[\left(u^{0}, v^{0}, p^{0}, F\right) \mathrm{e}^{\mathrm{i} t}+c . c .\right]+\varepsilon^{2}\left[\left(u^{\mathrm{s}}, t^{\mathrm{sE}}, p^{\varsigma}, f^{3}\right)+\cdots\right] \\
& +\varepsilon^{3}\left[\left(u_{1}^{0}, v_{1}^{0}, p_{1}^{0}, F_{1}\right) \mathrm{e}^{\mathrm{i} t}+\text { c.c. }+\cdots\right]+\cdots,
\end{aligned}
$$

where c.c. stands for the complex conjugate and only those terms that will be relevant below are displayed. Also, $F, F_{1}$ and all variables with superscripts o(=oscillatory), s(=steady, or time averaged) and/or $\mathrm{E}(=$ Eulerian $)$ are independent of $t$. Thus $\left(u^{\mathrm{s} \mathrm{E}}, v^{\mathrm{s} \mathrm{E}}\right)$ is a first approximation of the time-averaged velocity (an Eulerian mean). Substituting (2.8) into (2.1)-(2.6) we obtain at order $O(\varepsilon)$,

$$
\begin{aligned}
& u_{x}^{0}+v_{y}^{0}=\mathrm{i} u^{0}+p_{x}^{0}-1 / 2=\mathrm{i} v^{0}+p_{y}^{0}=0, \\
& v^{0}=0 \quad \text { at } y=-1, \quad u^{0}=0 \quad \text { at } x= \pm 1, \\
& v^{0}-\mathrm{i} f=p^{0}-G F+T F_{x x}=0 \quad \text { at } y=0, \\
& F=0 \quad \text { at } x= \pm 1,
\end{aligned}
$$

which uniquely determines $\left(u^{\circ}, v^{\circ}, p^{n}, F\right)$ provided that the oscillations are non-resonant (namely, that the homogeneous version of (2.9)-(2.12) has only the trivial solution) as we assume hereafter. In order to calculate $\left(u^{\mathrm{sE}}, v^{\mathrm{sE}}\right)$ we could think that vorticity vanishes if it is zero initially. This and the continuity equation (with inviscid boundary conditions) would give $u^{\mathrm{s}}=v^{\mathrm{s}}=0$, but this would be wrong because vorticity is non zero in the boundary layers and can (and will!) diffuse and be convected into the bulk. This job is to be taken by the mean flow, which is given in the bulk by the following equations:

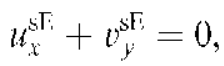

$$
\begin{aligned}
& \left(v^{\mathrm{s} \mathrm{E}}+v^{\mathrm{sc}}\right)\left(u_{y}^{\mathrm{s} \mathrm{E}}-v_{x}^{\mathrm{s} \mathrm{E}}\right)=-q_{x}^{\mathrm{s}}+R_{\mathrm{s}}^{-1}\left(u_{x x}^{\mathrm{s} \mathrm{E}}+u_{y y}^{\mathrm{s} \mathrm{E}}\right), \\
& -\left(u^{\mathrm{sF}}+u^{\mathrm{Sd}}\right)\left(u_{y}^{\mathrm{sF}}-v_{x}^{\mathrm{sE}}\right)=-q_{y}^{\mathrm{s}}+R_{\mathrm{s}}^{-1}\left(v_{x x}^{\mathrm{s}}+v_{y y}^{\mathrm{s}}\right),
\end{aligned}
$$

where $q^{8}$ is a modified averaged pressure at order $O\left(\varepsilon^{4}\right)$ and

$$
\left(u^{\mathrm{sd}}, v^{\mathrm{sil}}\right)=\mathrm{i}\left(\bar{u}^{0} u_{x}^{\mathrm{o}}+\bar{v}^{\mathrm{o}} u_{y}^{\mathrm{o}}, \bar{u}^{0} v_{x}^{0}+\bar{v}^{0} v_{y}^{\mathrm{o}}\right)+\text { c.c. }
$$


is the Stokes drift velocity. The overbar denotes hereafter the complex conjugate. Let us recall here that the Stokes drift appears in a natural way when calculating the mass transport velocity (a Lagrangian mean), which is given by (Batchelor, 1967)

$$
\left(u^{\mathrm{S}}, v^{\mathrm{s} \mathrm{L}}\right)=\left(u^{\mathrm{s}}, v^{\mathrm{sF}}\right)+\left(u^{\mathrm{Sd}}, v^{\mathrm{Sd}}\right)
$$

and is the velocity associated with the time-averaged trajectories of material elements (thus the appropriate one for, e.g. convection of passive scalars). The streaming flow Reynolds number is defined as

$$
R_{\mathrm{s}}=u^{2} R \equiv \omega c^{2} / v \equiv\left(a / t_{\mathrm{b}}\right)^{2}
$$

and is assumed to be of order one for simplicity (but it can be large, see below). Eq. (2.13) is obtained by substituting (2.8) into (2.1) and averaging in time. Eqs. (2.14) and (2.15) instead are obtained at order $\mathrm{O}\left(c^{4}\right)$ and those terms depending on the Stokes drift velocity are obtained after some algebra from the time average of the Reynolds stresses resulting form products of oscillatory terms at orders $O(\varepsilon)$ and $O\left(\varepsilon^{3}\right)$, namely

$$
\left(\bar{v}^{\circ}\left(u_{y}^{\circ}-v_{1 x}^{\circ}\right),-\bar{u}^{\circ}\left(u_{1 y}^{o}-v_{i_{x}}^{\circ}\right)\right)+\text { c.c. }
$$

where $u_{1}^{\circ}$ and $v_{1}^{\circ}$ satisfy

$$
\mathrm{i} u_{1}^{\mathrm{o}}+v^{\mathrm{o}}\left(u_{y}^{\mathrm{sF}}-v_{x}^{\mathrm{SF}}\right)+p_{\left.\right|_{x}}^{\mathrm{o}}=\mathrm{i} v_{1}^{\mathrm{o}}-u^{\mathrm{o}}\left(u_{y}^{\mathrm{sF}}-v_{x}^{\mathrm{SF}}\right)+p_{1 y}^{\mathrm{o}}=0 .
$$

Replacing (2.19) into (2.18) yields the required terms appearing in (2.14) and (2.15) plus a potential contribution to the modified pressure $q^{s}$. Note that the oscillatory terms at orders $O(z)$ and $O\left(\varepsilon^{2}\right)$ are potential. Thus their products yield zero Reynolds stresses.

Now, Eqs. (2.13)-(2.15) apply in the bulk (in fact, in the umperturbed bulk, bounded by the solid walls and the unperturbed free boundary) and must be accompanied by the following boundary conditions:

$$
\begin{aligned}
& u^{\mathrm{sE}}=-3\left[(1+\mathrm{i}) u^{\mathrm{0}} \bar{u}_{x}^{\mathrm{o}}+\text { c.c. }\right] / 2, \quad v^{\mathrm{sE}}=0 \quad \text { at } y=-1, \\
& u^{\mathrm{sE}}=0, \quad v^{\mathrm{sE}}=-3\left[(1+\mathrm{i}) v^{\mathrm{v}} \bar{v}_{y}^{\mathrm{v}}+\text { c.c. }\right] / 2 \quad \text { at } x= \pm 1, \\
& v^{\mathrm{sF}}=\left(\bar{F} u^{\mathrm{o}}\right)_{x}+\text { c.c. }, \quad u_{y}^{\mathrm{sE}}=2\left(\bar{F}_{u^{\mathrm{o}}}\right)_{x x}+4 \bar{F}_{x} u_{x}^{\mathrm{o}}+\text { c.c. }, \quad \text { at } y=0 .
\end{aligned}
$$

These result from imposing that the solution in the bulk matches with that in the boundary layers.

Let us first consider the Stokes boundary layer attached to the bottom of the container, which has a thickness $R^{-1 / 2}$ (or, in dimensional terms, $\ell_{\mathrm{bl}}$, defined above). Introducing the stretched variable

$$
\eta=R^{1 / 2}(y+1)
$$

and the expansions

$$
(u, v, p)=\varepsilon\left[\left(\check{u}^{\mathrm{o}}, R^{-1 / 2} \tilde{v}^{0}, \tilde{p}^{0}\right) \mathrm{c}^{\mathrm{i} t}+\mathrm{c.c} .\right]+\tilde{\iota}^{2}\left[\left(\tilde{u}^{\mathrm{s}}, R^{-1 / 2} \tilde{v}^{\varsigma}, \tilde{p}^{\mathrm{s}}\right)+\cdots\right]+\cdots,
$$


into (2.1)-(2.3), we obtain the following equations at orders $O(\varepsilon)$ and $O\left(\varepsilon^{2}\right)$

$$
\begin{aligned}
& \tilde{u}_{x}^{0}+\tilde{v}_{\eta}^{0}=0, \quad \mathrm{i} \tilde{u}^{0}=-\tilde{p}_{x}^{0}+\tilde{u}_{\eta \eta}^{0}+1 / 2, \quad \tilde{p}_{\eta}^{0}=0, \\
& \tilde{u}_{m}^{\mathrm{s}}-\tilde{p}_{x}^{\mathrm{s}}=\overline{\tilde{v}}^{0} \tilde{u}_{\eta}^{0}+\text { c.c., } \quad \tilde{p}_{\eta}^{\mathrm{s}}=\overline{\tilde{u}}^{0} \tilde{u}_{\eta}^{0}+\text { c.c. },
\end{aligned}
$$

with boundary conditions

$$
\tilde{u}^{\circ}=\tilde{v}^{\circ}=u^{s}=v^{s}=0 \quad \text { at } \eta=0, \quad \tilde{u}_{\eta}^{0}=\tilde{u}_{\eta}^{s}=0 \quad \text { as } \eta \rightarrow \infty,
$$

where we are anticipating a matching condition with the outer flow. Integration of (2.25)-(2.27) yields

$$
\tilde{u}^{0}=-U\left(1-\mathrm{e}^{-\sqrt{\mathrm{i} i} \eta}\right), \quad \tilde{v}^{v}=-U_{x}\left[\eta+\left(\mathrm{e}^{-\sqrt{\mathrm{i} \eta}}-1\right) / \sqrt{\mathrm{i}}\right), \quad \tilde{p}^{\mathrm{s}}=\left|\tilde{u}^{0}\right|^{2}+P,
$$

where $U$ and $P$ depend only on $x$, and

$$
\tilde{u}^{s}=-3\left[(1+\mathrm{i}) U \bar{U}_{x}+\text { c.c. }\right] / 2+\mathrm{O}\left(\mathrm{e}^{-\eta / \sqrt{ } 2}\right) \text { as } \eta \rightarrow \infty .
$$

And we only need to compare (2.8) with (2.24) using (2.28a and b) and (2.29), and require matching between both (which yield $U=u^{0}(x,-1)$ and $P=p^{0}(x,-1)$ ) to obtain the boundary conditions (2.20). The boundary conditions (2.21) are obtained from (2.20) using invariance under translation and rotation. The derivation of $(2.22)$ is omitted here but we must mention that (in addition to being much more involved) it bears an additional difficulty. Namely, since the free boundary vertical displacement can be large compared to the boundary layer thickness (which occurs if $R_{\mathrm{s}}$ is large, see (2.17)), we must use a time-dependent curvilinear coordinate system attached to the free boundary to analyze the boundary layer. Solving the equations in the boundary layer and applying matching conditions with the outer flow we obtain the time averaged normal velocity $\left(\simeq v^{\mathrm{s} \Sigma}\right)$ and shear stress ( $\tau^{\mathrm{sE}} \simeq \partial u^{\mathrm{s} E} / \partial y$ ) of the outer flow at the edge of the boundary layer, which is $y=f$ in first approximation; and these conditions must be translated to the unperturbed free boundary $y=0$ using a Taylor expansion (recall that $f$ is small).

The analysis above does not apply near the corners at $x= \pm 1, y=-1,0$, where some small viscous regions appear that should be in principle analyzed separately. But an orders-of-magnitude estimate readily shows that these regions only produce a higher order effect on the mean flow in the bulk because the Reynolds stresses are much smaller there than in the oscillatory boundary layers. It was precisely because of this that we pinned the triple points $(x= \pm 1, y=0)$ to the upper edge of the lateral walls. Note nevertheless that if the triple points were allowed to oscillate with an unperturbed contact angle different from 0 and $\pi$, then the Reynolds stresses would be huge in these regions, which could have a net effect on the streaming flow in the bulk. But unfortunately these regions have not been analyzed for oscillating triple points, which would be a non-trivial task (in fact, a generalization of the classical work by Moffatt, 1964).

The boundary conditions $(2.20)-(2.21)$ are readily extended to curved smooth boundaries by just replacing $u^{\circ}$ or $v^{\circ}$ by the tangential velocity components, and $x$ or $y$ by an are length parameter (Batchelor, 1967). A similar extension of (2.22) includes some new terms that depend on the curvature of the unperturbed free boundary (Longuet-Higgins, 1953) (see (5.32) below) and are due to the vibrating motion of the free boundary. And the same happens near vibrating deformable solids, see Section 6 below. The extension of these boundary conditions to $3 \mathrm{D}$ requires more care. If the solid boundary or the unperturbed free boundary is a plane, then the tangential velocity components 
will be vectors and the tangential derivatives should be replaced in (2.20)-(2.22) by 2D projections on the plane of the 3D $\nabla$ operator, $\tilde{\nabla}$. But the final formulac cannot be guessed. For instance, a second derivative of a vector $v$ could lead to, e.g. either the (2D) Laplacian of $v$ or $\tilde{\nabla}(\tilde{\nabla} \cdot v)$. This extension has already been made by Hunt and Johns (1963) and Liu (1977). If, in addition, the solid boundary or unperturbed free boundary is a more general smooth surface, then we must use the projection of the velocities on the tangent plane and the intrinsic gradien operator along the surface, and the results will depend on the (principal) curvature(s) of the surface. This generalization requires some well-known (Aris, 1962) tools from Differential Geometry, which are systematically used in related contexts (Romano, 1993).

\section{Formulation of the $3 \mathrm{D}$ problem}

Let us consider a liquid occupying a region of the 3D space, bounded by a (possibly deformable) solid and/or a free boundary; e.g. a bubble, a pendant drop, a capillary bridge or a liquid-filled container. The solid boundary (if present) and/or the reference frame are/is oscillating harmonically with a frequency $a$. As in Section 2 we use $\omega^{-1}$ and $t$ as characteristic time and length for non-dimensionalization, where $f$ is required to be of the order of the wavelength of the excited oscillatory flow. The continuity and Navier-Stokes equations are

$$
\boldsymbol{\nabla} \cdot \boldsymbol{v}=0, \quad \hat{\mathrm{v}} / \hat{\mathrm{o} t}+(\boldsymbol{v} \cdot \boldsymbol{\nabla}) \mathbf{v}=-\boldsymbol{\nabla} p+R^{-1} \Delta \boldsymbol{v}+\mathrm{r} \boldsymbol{a} \cos t
$$

where $\boldsymbol{v}$ is the velocity, $p$ is the hydrostatic pressure and $\boldsymbol{a}$ is a fixed unit vector. For convenience we are not decomposing here convective terms into Reynolds stresses and a potential part, as we did in Section 2. These equations are subject to the following boundary conditions

$$
\begin{aligned}
& \boldsymbol{v}=\boldsymbol{v}^{w} \equiv \varepsilon \boldsymbol{V}^{w^{\prime}} \mathrm{c}^{\mathrm{i} /}+\mathrm{c.c} \text {. at the no slip boundary, } \\
& \boldsymbol{v} \cdot \boldsymbol{n}_{F}=(\partial f / \partial t) \boldsymbol{n} \cdot \boldsymbol{n}_{F}, \quad\left[\left(\nabla \boldsymbol{v}+\boldsymbol{\nabla} \boldsymbol{v}^{\top}\right) \cdot \mathbf{n}_{F^{\top}}\right] \times \boldsymbol{n}_{F^{-}}=\mathbf{0} . \\
& p-G n_{F} \cdot \mathbf{e}_{z} f+2 T M(f)=R^{-1}\left[\left(\nabla v+\nabla v^{\top}\right) \cdot \mathbf{n}_{F}\right] \cdot \mathbf{n}_{F} \quad \text { at the free boundary. }
\end{aligned}
$$

Here $V^{w}$ is a given vector function of position along the boundary, $e_{z}$ is the upward unit vertical vector, $\boldsymbol{n}_{F}$ and $\boldsymbol{n}$ are the outward unit normals to the free boundary and the unperturbed free boundary, respectively, $f$ is the free boundary deflection along $n, M(f)$ is the mean curvature of the free boundary, and the superscript $T$ stands for the transpose. The parameters $\varepsilon, G, T$, and $R$ are defined as in Section 2 and again required to satisfy (2.7), which is the basic assumption and is rewritten here for convenience

$$
\varepsilon=a / t \ll 1, \quad R=\omega t^{2} / v \equiv\left(\ell / t_{\mathrm{bl}}\right)^{2} \gg 1,
$$

where $t_{\mathrm{bl}}=\sqrt{v / \omega}$ is the thickness of the oscillatory boundary layers. The expansions (2.8) are now

$$
(\boldsymbol{v}, p, f)=\varepsilon\left(\left(\boldsymbol{u}^{\mathrm{o}}, p^{0}, F\right) \mathrm{e}^{\mathrm{i} t}+\text { c.c. }\right)+\varepsilon^{2}\left(\left(\boldsymbol{v}^{\mathrm{sF}}, p^{\mathrm{s}}, f^{\mathrm{s}}\right)+\cdots\right)+\cdots,
$$

where $\boldsymbol{u}^{\mathrm{O}}$ and $\boldsymbol{v}^{\mathrm{sE}}$ (and their counterparts for $p$ and $f$ ) are independent of time and $v^{\mathrm{E}}$ is the (Eulerian) time-averaged velocity. The counterpart of the problem (2.9)-(2.12) giving the oscillatory 
flow in the bulk is now

$$
\begin{aligned}
& \nabla \cdot \boldsymbol{u}^{\circ}=0, \quad \mathrm{i} \boldsymbol{u}^{\circ}+\nabla p^{0}-\boldsymbol{a} / 2=\mathbf{0}, \\
& \boldsymbol{u}^{\circ} \cdot \mathbf{n}=\boldsymbol{V}^{w} \cdot \mathbf{n} \quad \text { at the unperturbed no slip boundary, } \\
& \boldsymbol{u}^{\circ} \cdot \mathbf{n}=\mathrm{i} F, \quad p^{0}-G \boldsymbol{n} \cdot \boldsymbol{e}_{z} F+T \tilde{\Delta} F=0 \text { at the unperturbed free boundary, }
\end{aligned}
$$

where $\tilde{\Delta}$ is the intrinsic Laplacian operator along the unperturbed boundary (u.b.). As in Section 2 we assume that the homogeneous version of (3.6)-(3.8) has only the trivial solution; otherwise, the solution depends on arbitrary complex constants (or amplitudes) whose calculation requires to also derive amplitude equations, involving higher order (viscous and/or non-linear) terms (Nicolás and Vega, 1996; Vega et al., 2001; Martin et al., 2002). The unique solution to this inviscid, linear problem can be obtained upon separation of variables for appropriate geometries (or numerically otherwise) and will be considered below as known. And the counterparts of the mean flow equations (2.13)-(2.15) are

$$
\begin{aligned}
& \boldsymbol{\nabla} \cdot \boldsymbol{v}^{\mathrm{sE}}=0, \\
& -\left(\boldsymbol{v}^{\mathrm{sE}}+\boldsymbol{v}^{\mathrm{Sd}}\right) \times\left(\boldsymbol{\nabla} \times \boldsymbol{v}^{\mathrm{sE}}\right)=-\nabla p^{\mathrm{s}}+R_{\mathrm{s}}^{-1} \Delta \boldsymbol{v}^{\mathrm{sE},}
\end{aligned}
$$

where the streaming flow Reynolds number (which as in Section 2 can be large) is defined as in Section 2, namely

$$
R_{\mathrm{S}}=\varepsilon^{2} R \equiv \omega \alpha^{2} / v \equiv\left(a / \ell_{\mathrm{bl}}\right)^{2},
$$

and the Stokes drift velocity is now given by

$$
\boldsymbol{v}^{\mathrm{sd}}=\mathrm{i}\left(\bar{u}^{\mathrm{o}} \cdot \nabla\right) \boldsymbol{u}^{\mathrm{o}}+\mathrm{c} . \mathrm{c} . \equiv \mathrm{i} \nabla \times\left(\boldsymbol{u}^{\mathrm{o}} \times \bar{u}^{\mathrm{o}}\right) \text {. }
$$

The boundary conditions for the streaming flow (namely, the counterparts of $(2.20)-(2.22)$ ) are

$$
\begin{aligned}
& \tilde{\boldsymbol{v}}^{\mathrm{F}}=\boldsymbol{\Phi}\left(\boldsymbol{U}, \boldsymbol{V}^{w}\right), \quad \boldsymbol{v}^{\mathrm{sE}} \cdot \mathbf{n}=\phi\left(\boldsymbol{U}, \boldsymbol{V}^{w}\right) \text { at the unperturbed solid boundary, } \\
& \tilde{\tau}^{\mathrm{sE}}=\boldsymbol{\Phi}(\boldsymbol{U}, F), \quad \boldsymbol{v}^{\mathrm{sE}} \cdot \mathbf{n}=\phi(\boldsymbol{U}, F) \text { at the unperturbed free boundary, }
\end{aligned}
$$

where the tilde over a vector denotes the orthogonal projection of the vector over the tangent plane to the u.b.; in particular, $\tilde{\tau}^{\mathrm{sF}}$ is the Eulerian shear stress, namely the orthogonal projection of the Eulerian stress $\tau^{\mathrm{sF}} \equiv\left[\nabla\left(v^{\mathrm{sF}}\right)+\nabla\left(v^{\mathrm{sF}}\right)^{\top}\right] \cdot \mathbf{n}$. The right-hand sides, $\Phi$ and $\phi$, will be calculated in Sections 4-6, in terms of $\boldsymbol{U}=\tilde{\boldsymbol{u}}^{\text {c }}$ (u.b.), $\boldsymbol{V}^{w}$, and $F$, see (3.7) and (3.8). This will require to apply matching conditions between the solutions in the bulk and in the oscillatory boundary layers.

Although the analysis above was made for $R_{s} \sim 1$, it remains valid under the sole assumptions (3.4), which are the only assumptions in this paper. Note that under $(3.4) R_{\mathrm{s}}$ varies in a wide range, from small to large values, because $R^{-1}$ and $\varepsilon$ are independent small parameters. If $R_{\mathrm{s}} \gg 1$, then the mean flow exhibits a secondary viscous boundary layer on top of the primary oscillatory boundary layers considered above (Stuart, 1966).

Once the linear problem (3.6)-(3.8) is solved, which is usually a reasonable task, the right-hand sides of (3.10), (3.13) and (3.14) are readily calculated (the functions $\Phi$ and $\phi$ will be obtained below), and the non-linea problem (3.9)-(3.10), (3.13) and (3.14) can be solved, numerically except 
for small $R_{\mathrm{s}}$ and appropriate geomctries. Note that the streaming flow equations are decoupled from the steady deflection of the free boundary, $f^{s}$, which can be calculated a posteriori from $(3.3)$.

The analysis in Sections 4-6 will require to solve the equations of motion in the oscillatory boundary layers. The flow near vibrating solid walls and free boundaries is conveniently analyzed in a frame attached to the u.b. To this end, at each normal to the u.b, the velocity is decomposed into its tangential and normal components as

$$
\tilde{\boldsymbol{v}}=\boldsymbol{v}-\tilde{w} \boldsymbol{n} \text { and } \tilde{w}=\boldsymbol{v} \cdot \boldsymbol{n} \text {. }
$$

Using the formulae in Appendix A, the governing Eqs. (3.1) are rewritten as

$$
\begin{aligned}
& \tilde{\nabla} \cdot \tilde{v}+\hat{w} \tilde{w} / \hat{\xi}-2 M \tilde{w}+O(|\tilde{v}|+|\tilde{w}|)|\xi|=0, \\
& \partial \tilde{\boldsymbol{v}} / \partial t+(\tilde{\boldsymbol{v}} \cdot \tilde{\nabla}) \tilde{\boldsymbol{v}}+\tilde{w} \partial \tilde{\mathbf{v}} / \partial \xi-\tilde{w} \mathscr{L} \tilde{\mathbf{v}}=-\tilde{\nabla} p-\xi \mathscr{L}(\tilde{\nabla} p)+R^{-1} \partial^{2} \tilde{\boldsymbol{v}} / \partial \xi^{2} \\
& +\mathrm{O}\left(\left(|\tilde{\boldsymbol{v}}|^{2}+|\tilde{w}|^{2}+|p \xi|\right)|\xi|+(|\tilde{\mathbf{v}}|+|\tilde{w}|) R^{-1}\right) \text {, } \\
& \partial \tilde{w} / \partial t+\tilde{w} \partial \tilde{w} / \partial \xi=-\partial p / \hat{\partial}+R^{-1} \hat{\partial}^{2} \tilde{w} / \partial \xi^{2}+O\left(\left(|\tilde{v}|^{2}+|\tilde{w}|^{2}\right)+(|\tilde{\boldsymbol{v}}|+|\tilde{w}|) R^{-1}\right),
\end{aligned}
$$

where we are anticipating that in the boundary layers considered below $|\tilde{\nabla}| \sim 1$ and $|\partial / \partial \xi| \sim R^{1 / 2}$. Here, $\zeta$ is a coordinate along $n, \tilde{\nabla}$. and $\tilde{\nabla}$ are the intrinsic divergence and gradient operators along the u.b., and $\mathscr{L}$ is the Weingarten map of the u.b., which bears complete information on the curvature of the u.b.. Let us recall here that the Weingarten map (Thorpe, 1979) of an oriented surface $S$, with a unit normal $n$, is defined as $\mathscr{L} \tilde{v}=-(\tilde{\boldsymbol{v}} \cdot \tilde{\nabla}) n$. This map is associated with the second fundamental form of the surface by $I I(\tilde{v})=\mathscr{L}(\tilde{v}) \cdot \tilde{v}$. Thus the curvature of the normal section of $S$ that is tangent to the unit vector $\tilde{\boldsymbol{v}}$ is given by $\kappa_{n}=\mathscr{L}(\tilde{\boldsymbol{v}}) \cdot \tilde{\boldsymbol{v}}$. Still, the eigenvalues of $\mathscr{L}$ are the principal curvatures and the associated eigenvectors are tangent to the curvature directions. Thus we have

$$
\mathscr{L}\left(v_{1} e_{1}+v_{2} e_{2}\right)=\kappa_{1} v_{1} e_{1}+\kappa_{2} v_{2} e_{2}
$$

where $e_{1}$ and $\boldsymbol{c}_{2}$ are unit vectors along two curvature directions, and $\kappa_{1}$ and $\kappa_{2}$ are the associated principal curvatures.

Similarly, if $f$ is the (small) deflection of the free boundary along $\boldsymbol{n}$ then the boundary conditions $(3.3 \mathrm{a}$ and $\mathrm{b})$ are rewritten as

$$
\begin{aligned}
& \tilde{w}=\hat{\jmath} f / \partial t+\tilde{\mathbf{v}} \cdot \tilde{\nabla} f+\mathrm{O}\left((|\tilde{\mathbf{v}}|+|\tilde{w}|)|f|^{2}\right), \\
& \hat{\partial} \tilde{\boldsymbol{v}} / \partial \dot{\mathcal{L}}+\mathscr{L} \tilde{\boldsymbol{v}}+\tilde{\nabla} \tilde{w}+f \mathscr{L}(\mathscr{L} \tilde{\boldsymbol{v}}+\tilde{\nabla} \tilde{w})-\left(\tilde{\nabla} \tilde{\boldsymbol{v}}+\tilde{\nabla} \tilde{\boldsymbol{v}}^{\top}\right) \cdot \tilde{\nabla} f \\
& +2(\partial \tilde{w} / \partial \xi) \tilde{\nabla} f+2 \tilde{w} \mathscr{L}(\tilde{\nabla} f)=\mathrm{O}\left((|\tilde{v}|+|\tilde{w}|)|f|^{2}\right) \text { at } \xi=f .
\end{aligned}
$$

These equations and boundary conditions are of independent interest in, c.g. the analysis of the flow in thin films (Couder et al., 1989) of arbitrary shape.

\section{The boundary layer attached to a static solid wall}

As in the analysis of the Stokes boundary layer in Section 2 (except for a change in sign), we use the stretched coordinate

$$
\eta=R^{1 / 2} \xi
$$


and seek the following expansions

$$
\begin{aligned}
& (p, \tilde{\boldsymbol{v}})=c(P, \boldsymbol{V}) \mathrm{c}^{\mathrm{i} t}+\mathrm{c.c} .+\varepsilon^{2}\left[\left(p^{\mathrm{s}}, \boldsymbol{v}^{\mathrm{s}}\right)+\mathrm{OT}\right]+\cdots, \\
& \tilde{w}=\varepsilon R^{-1 / 2} W \mathrm{e}^{\mathrm{i} t}+\text { c.c. }+\varepsilon^{2}\left(w^{\mathrm{s}}+\mathrm{OT}\right)+\cdots,
\end{aligned}
$$

where $V, v^{s}, W, w^{s}, P$, and $p^{s}$ are time independent and $O \mathrm{~T}$ stands for oscillatory terms, depending on $l$ as $\mathrm{e}^{\mathrm{i} m t}$, with $m \neq 0$. Note that $\varepsilon^{2} v^{s}$ and $\varepsilon^{2} u^{\mathrm{s}}$ are the steady parts of the velocity components at leading order. Replacing $(4.1)-(4.2)$ into $(3.16)-(3.18)$ we obtain the following equations at orders $\varepsilon$ and $\varepsilon^{2}$

$$
\begin{aligned}
& \partial^{2} \boldsymbol{V} / \partial \eta^{2}-\mathrm{i} \boldsymbol{V}=\tilde{\nabla} P, \quad \partial P / \partial \eta=0, \quad \partial W / \partial \eta=-\tilde{\boldsymbol{\nabla}} \cdot \mathbf{V}, \\
& \partial^{2} \boldsymbol{v}^{\mathrm{s}} / \partial \eta^{2}-\tilde{\nabla} p^{s}=(\overline{\boldsymbol{V}} \cdot \tilde{\boldsymbol{\nabla}}) \boldsymbol{V}+\bar{W} \partial \boldsymbol{V} / \hat{\imath} \eta+c \cdot c \cdot \quad \hat{\partial} p^{\mathrm{s}} / \partial \eta=\partial w^{\mathrm{s}} / \partial \eta=0,
\end{aligned}
$$

in $-\infty<\eta<0$. Also, Eq. (3.2) and matching conditions with the outer flow lead to

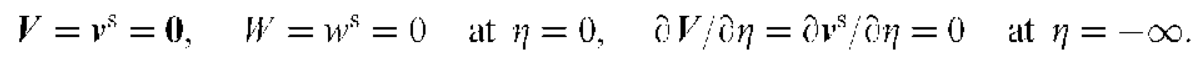

Integration of (4.3)-(4.5) subsequently yields

$$
\boldsymbol{V}=\boldsymbol{U}\left(1-\mathrm{e}^{\sqrt{\mathrm{i} \eta})}, \quad \boldsymbol{W}=-\left[\eta+\left(1-\mathrm{e}^{\sqrt{\mathrm{i} \eta}}\right) / \sqrt{\mathrm{i}}\right] \tilde{\nabla} \cdot \mathbf{U},\right.
$$

in $-\infty<\eta<0$, where as defined above $U$ is such that the tangential component of the outer flow at the solid wall is given by $\tilde{\boldsymbol{v}}=c\left(\boldsymbol{U} \mathrm{c}^{\mathrm{i} /}+\mathrm{c} . \mathrm{c}.\right)+O\left(\varepsilon^{2}\right)$ and

$$
\boldsymbol{v}^{\mathrm{s}}=\boldsymbol{\Phi} \equiv-[(2+3 \mathrm{i})(\tilde{\boldsymbol{\nabla}} \cdot \overline{\boldsymbol{U}}) \boldsymbol{U}+(\overline{\boldsymbol{U}} \cdot \tilde{\nabla}) \boldsymbol{U}+\text { c.c. }] / 2, \quad w^{\mathrm{s}}=\phi \equiv 0,
$$

at $\eta=-\infty$. Now, we only need to apply matching conditions with the outer, inviscid flow, invoking (3.5) and (4.2), to obtain that these expressions for $\Phi$ and $\phi$ are precisely the right-hand sides of (3.13). And using this, (3.12) and (A.9), the tangential and normal components of the Lagrangian mean tangential velocity, $v^{\mathrm{sL}}=v^{\mathrm{sE}}+v^{\mathrm{sd}}$, at the edge of the boundary layer are found to be (cf. $(2.20)-(2.21))$

$$
\tilde{\boldsymbol{v}}^{\mathrm{s}}=-[(2+3 \mathrm{i})(\tilde{\nabla} \cdot \overline{\boldsymbol{U}}) \boldsymbol{U}+(1-2 \mathrm{i})(\overline{\boldsymbol{U}} \cdot \tilde{\nabla}) \boldsymbol{U}+\mathrm{c.c} .] 2, \quad \boldsymbol{v}^{\mathrm{s}} \cdot \mathbf{n}=0 .
$$

As in Section 2 the forcing tangential velocity is independent of both viscosity (i.e., of $R$ ) and the curvature of the boundary. The latter will not be true for vibrating boundaries (Sections $5-6$ below).

These formulae generalize the well known ones in 2D (Batchelor, 1967), namely (cf. (2.20)(2.21))

$$
u^{\mathrm{s} s}=-3[(1+\mathrm{i}) U \mathrm{~d} \bar{U} / \mathrm{d} s+\mathrm{c.c} .] / 2, \quad u^{\mathrm{s} s}=-[(3+5 i) U \mathrm{~d} \bar{U} / \mathrm{d} s+\mathrm{c} . \mathrm{c} .] / 2 .
$$

Here, $\tilde{v}$ and $\boldsymbol{U}$ have been written as $\tilde{v}=u t$ and $\boldsymbol{U}=U \boldsymbol{t}$, where $\boldsymbol{t}$ is a unit tangent vector to the u.b. and $s$ is an arc length parameter along the u.b.

\section{Oscillatory boundary layer attached to a free boundary}

Now the boundary layer is oscillating with the free boundary. As explained at the end of Section 2, the derivation of the boundary conditions (3.14) is made in two steps: in Section 5.1 we analyze the oscillatory boundary layer in a reference frame attached to the free boundary, and apply matching 
conditions with the outer flow at the edge of this layer; and in Section 5.2 we obtain the boundary conditions of the outer flow at the unperturbed free boundary.

\subsection{The oscillatory boundary layer}

We redefine the normal velocity component and use the following stretched coordinate attached to the free boundary

$$
\tilde{w}^{*}=\tilde{w}-\partial f / \partial t-\tilde{v} \cdot \tilde{\nabla} f, \quad \eta=R^{1 / 2}(\xi-f),
$$

to rewrite Eqs. (3.16)-(3.18) as

$$
\begin{aligned}
& \partial \tilde{w}^{*} / \partial \eta+R^{-1 / 2}(\tilde{\nabla} \cdot \tilde{v}-2 M \hat{\partial} f / \partial t)=O\left(\varepsilon^{2} R^{-1 / 2}+\varepsilon R^{-1}\right), \\
& \hat{\partial \boldsymbol{v}} / \hat{\partial} t+(\tilde{\boldsymbol{v}} \cdot \tilde{\boldsymbol{\nabla}}) \tilde{\boldsymbol{v}}+R^{1 / 2}\left[\left(\tilde{w}^{*}+\tilde{\boldsymbol{v}} \cdot \tilde{\boldsymbol{\nabla}} f\right) \hat{\mathrm{v}} / \hat{\jmath} \eta-(\hat{\jmath} p / \hat{\jmath} \eta \tilde{\boldsymbol{\nabla}} f]-(\partial f / \partial t) \mathscr{L} \tilde{\boldsymbol{v}}+\tilde{\boldsymbol{\nabla}} p\right. \\
& +\left(f+R^{-1 / 2} \eta\right) \mathscr{L}(\tilde{\nabla} p)-\partial^{2} \tilde{\boldsymbol{v}} / \widehat{\iota} \eta^{2}=\mathrm{O}\left(\varepsilon\left(\varepsilon+R^{-1 / 2}\right)^{2}\right), \\
& \hat{\partial} p / \partial \eta+R^{-1 / 2}\left(\hat{\partial}^{2} f / \partial t^{2}-\partial^{2} \tilde{w}^{*} / \partial \eta^{2}\right)=\mathrm{O}\left(\hat{\imath}\left(\varepsilon+R^{-1 / 2}\right)^{2}\right)
\end{aligned}
$$

where we are anticipating that the boundary layer thickness is $O\left(R^{-1 / 2}\right)$ and that $|\tilde{v}| \sim|f| \sim \varepsilon$ and $\left|\tilde{w}^{*}\right| \sim \varepsilon R^{-1 / 2}+\varepsilon^{2}$. Note that these equations do not coincide with the original Eqs. (3.16)(3.18); thus the Prandtl transposition theorem (which only applies at leading order and requires that $|f| \sim R^{-1 / 2}$, Glauert, 1957) cannot be applicd here. Similarly, the boundary conditions (3.20)-(3.21) are rewritten as

$$
\begin{aligned}
& \tilde{w}^{*}=O\left(\varepsilon^{3}+\varepsilon^{2} R^{-1 / 2}\right) \\
& \partial \tilde{v} / \partial \eta+\left(\partial \tilde{w}^{*} / \partial \eta\right) \tilde{\nabla} f+R^{-1 / 2}[\mathscr{L} \tilde{v}+\tilde{\nabla}(\partial f / \partial t+\tilde{v} \cdot \tilde{\nabla} f)] \\
& \quad+R^{-1 / 2}[f \mathscr{L}(\mathscr{L} \tilde{\mathbf{v}}+\tilde{\nabla}(\partial f / \partial t))+2(\partial f / \partial t) \mathscr{L}(\tilde{\nabla} f)] \\
& \quad-R^{-1 / 2}\left(\tilde{\nabla} \tilde{\mathbf{v}}+\tilde{\nabla} \tilde{v}^{\top}\right) \cdot \tilde{\nabla} f=O\left(\varepsilon^{3}+\imath R^{-1}\right) \quad \text { at } \eta=0 .
\end{aligned}
$$

Now we seek the expansions

$$
\begin{aligned}
& f=c F \mathrm{c}^{\mathrm{i} t}+\text { c.c. }+\cdots, \\
& \tilde{v}=\varepsilon\left(\boldsymbol{V}_{0}+R^{-1 / 2} \boldsymbol{V}_{1}\right) \mathrm{e}^{\mathrm{i} t}+\text { c.c. }+\varepsilon^{2}\left(\boldsymbol{v}_{2}^{\mathrm{s}}+R^{-1 / 2} \boldsymbol{v}_{3}^{\mathrm{s}}+\mathrm{OT}\right)+\cdots, \\
& \tilde{w}^{*}=\varepsilon R^{-1 / 2} W_{1} \mathrm{e}^{\mathrm{i} t}+\text { c.c. }+\varepsilon^{2}\left(w_{2}^{\mathrm{s}}+\mathrm{OT}\right)+\cdots, \\
& p=\varepsilon\left(P_{0}+R^{-1 / 2} P_{1}\right) \mathrm{e}^{\mathrm{i} t}+\text { c.c. }+\varepsilon^{2}\left(p_{2}^{\varsigma}+R^{-1 / 2} p_{3}^{\mathrm{s}}+\mathrm{OT}\right)+\cdots,
\end{aligned}
$$


where the various coefficients $\left(F, V_{0}, V_{1}, \ldots\right)$ are independent of $\ell$. Substituting (5.7) into (5.2)-(5.4), the following equations result at orders $\varepsilon, \varepsilon R^{-1 / 2}$ and $\varepsilon^{2}$

$$
\begin{aligned}
& \hat{\partial}^{2} \boldsymbol{V}_{0} / \partial \eta^{2}-\mathrm{i} \boldsymbol{V}_{0}=\tilde{\nabla} P_{0}, \quad \partial P_{0} / \hat{\jmath}=0, \quad \partial W_{1} / \partial \eta=-\tilde{\nabla} \cdot \boldsymbol{V}_{0}+2 \mathrm{i} M F, \\
& \hat{\partial}^{2} V_{\mathrm{I}} / \partial \eta^{2}-\mathrm{i} V_{\mathrm{I}}=\tilde{\nabla} P_{1}+\eta \mathscr{L}\left(\tilde{\nabla} P_{0}\right), \quad \partial P_{\mathrm{I}} / \hat{\eta} \eta=F, \\
& \partial^{2} \boldsymbol{v}_{2}^{\mathrm{s}} / \partial \eta^{2}-\tilde{\nabla} p_{2}^{\mathrm{s}}=\bar{F} \mathscr{L}\left(\mathrm{i} \boldsymbol{V}_{0}+\tilde{\nabla} P_{0}\right)-\left(\hat{\partial} P_{1} / \hat{\partial}\right) \tilde{\nabla} \bar{F}+\left(\overline{\boldsymbol{V}}_{0} \cdot \tilde{\nabla}\right) \boldsymbol{V}_{0} \\
& +\bar{W}_{1} \partial \boldsymbol{V}_{0} / \partial \eta+\mathrm{c.c.}, \quad \partial p_{2}^{s} / \partial \eta=\partial w_{2}^{s} / \partial \eta=0 .
\end{aligned}
$$

in $-\infty<\eta<0$. We also impose the boundary conditions $(5.5)-(5.6)$, which lead to

$$
\begin{aligned}
& W_{1}=0, \quad \partial \boldsymbol{V}_{0} / \partial \eta=\mathbf{0}, \quad \partial \boldsymbol{V}_{1} / \partial \eta=-\left(\mathscr{L} \boldsymbol{V}_{0}+\mathrm{i} \tilde{\nabla} F\right), \\
& w_{2}^{s}=0, \quad \partial \boldsymbol{v}_{2}^{\mathrm{s}} / \partial \eta=\mathbf{0} \quad \text { at } \eta=0,
\end{aligned}
$$

and anticipate the following matching conditions with the outer flow

$$
\partial \boldsymbol{V}_{0} / \partial \eta=\partial^{2} \boldsymbol{V}_{1} / \partial \eta^{2}=\partial \boldsymbol{v}_{2}^{\mathrm{s}} / \partial \eta=\mathbf{0} \quad \text { as } \eta \rightarrow-\infty .
$$

Integration of $(5.8)-(5.10)$ yiclds

$$
\begin{aligned}
& P_{0}=P_{0}^{0}, \quad \boldsymbol{V}_{0}=\boldsymbol{U} \equiv \mathrm{i} \tilde{\nabla} P_{0}^{0}, \quad W_{1}=(2 \mathrm{i} M F-\tilde{\nabla} \cdot \mathbf{U}) \eta, \\
& P_{1}=F \eta+P_{1}^{0}, \quad \boldsymbol{V}_{1}=-2 \mathrm{c}^{\sqrt{\mathrm{i} \eta}} \boldsymbol{H} / \sqrt{\mathrm{i}}+\eta \boldsymbol{H}+\mathrm{i} \tilde{\nabla} P_{1}^{0}, \\
& w_{2}^{\mathrm{s}}=0, \quad \boldsymbol{v}_{2}^{\mathrm{s}}=\boldsymbol{v}_{2}^{\mathrm{s} 0}, \quad p_{2}^{\mathrm{s}}=-|\boldsymbol{U}|^{2}+|F|^{2}+p_{2}^{\mathrm{s} 0},
\end{aligned}
$$

where $U, P_{0}^{0}, P_{1}^{0}, p_{2}^{s 0}$ and $\boldsymbol{v}_{2}^{\mathrm{s} 0}$ are independent of $\eta$ and

$$
\boldsymbol{H}=\mathscr{L} \boldsymbol{U}+\mathrm{i} \tilde{\nabla} F \text {. }
$$

Now, using (5.11)-(5.13) the stcady part of the momentum Eqs. (5.3) and (5.4) and the boundary conditions (5.6) at order $\varepsilon^{2} R^{-1 / 2}$ can be written as

$$
\begin{aligned}
& \partial p_{3}^{\mathrm{s}} / \partial \eta=\mathrm{POL}, \\
& \partial^{2} \boldsymbol{v}_{3}^{\mathrm{s}} / \partial \eta^{2}=-2 \mathrm{c}^{\sqrt{\mathrm{i} \eta}}[(\boldsymbol{H} \cdot \tilde{\nabla}) \overline{\boldsymbol{U}}+(\overline{\boldsymbol{U}} \cdot \dot{\nabla}) \boldsymbol{H}+\mathrm{i} \bar{F} \mathscr{L} \boldsymbol{H}] / \sqrt{\mathrm{i}} \\
&+2 \eta \mathrm{c}^{\sqrt{\mathrm{i} \eta}}(2 \mathrm{i} M \bar{F}+\tilde{\nabla} \cdot \overline{\boldsymbol{U}}) \boldsymbol{H}+\mathrm{c.c}+\left(\tilde{\boldsymbol{\nabla}} p_{3}^{\mathrm{s}}+\mathrm{POL}\right)
\end{aligned}
$$

in $-\infty<\eta<0$, and

$$
\begin{aligned}
\partial \nu_{3}^{\mathrm{s}} / \partial \eta+\mathscr{L} \boldsymbol{v}_{2}^{\mathrm{s} \theta}= & \left(\tilde{\nabla} \boldsymbol{U}+\tilde{\nabla} \boldsymbol{U}^{\top}\right) \cdot \dot{\nabla} \bar{F}-\tilde{\nabla}(\boldsymbol{U} \cdot \tilde{\nabla} \bar{F}) \\
& -(2 \mathrm{i} M F-\tilde{\nabla} \cdot \mathbf{U}) \tilde{\nabla} \bar{F}-\bar{F} \mathscr{L}(\mathscr{L} \boldsymbol{U}-\mathrm{i} \tilde{\nabla} F)+\text { c.c. }
\end{aligned}
$$

at $\eta=0$, where POL stands for a polynomial in the $\eta$ variable, whose coeflicients can depend on position along the free boundary. Here we take into account that those terms of the order of $\varepsilon^{2} R^{-1 / 2}$, not displayed in (5.3), depend quadratically on $(\tilde{v}, \tilde{w}, p, f)$, and thus can only contribute to POL in (5.16). Also we anticipate a part of the matching conditions with the outer llow, namely $\hat{o}^{2} v_{3}^{3} / \delta \eta^{2}=0$ 
as $\eta \rightarrow-\infty$. Thus the right-hand side of (5.16) must vanish at $\eta=-\infty$ and, consequently, the last term there (i.e., $\tilde{\nabla} p_{3}^{s}+$ POL, which is a polynomial in $\eta$ because $p_{3}^{8}=$ POL, see (5.15)) identically vanishes. Then we only need to integrate (5.16) and take into account (5.17) to obtain

$$
\begin{aligned}
& \left(\partial v_{3}^{s} / \partial \eta\right)_{\eta}-\infty+\mathscr{L} v_{2}^{s 0}=-2 \mathrm{i}[(\boldsymbol{H} \cdot \tilde{\nabla}) \bar{U}+(\overline{\boldsymbol{U}} \cdot \tilde{\nabla}) \boldsymbol{H}+(\tilde{\nabla} \cdot \bar{U}) \boldsymbol{H}] \\
& +\bar{F} \mathscr{L} H+4 M \bar{F} H+\left(\dot{\nabla} \boldsymbol{U}+\tilde{\nabla} U^{\top}\right) \cdot \tilde{\nabla} \bar{F}-\tilde{\nabla}(\boldsymbol{U} \cdot \tilde{\nabla} \bar{F}) \\
& -(2 \mathrm{i} M F-\dot{\nabla} \cdot \mathrm{U}) \dot{\nabla} \bar{F}+2 \mathrm{i} \bar{F} \mathscr{L}(\tilde{\nabla} F)+\text { c.c. }
\end{aligned}
$$

Now we consider the tangential and normal velocity components of the outer flow. Invoking (5.1), $(5.7),(5.11)-(5.13)$, and $(5.18)$ we obtain

$$
\begin{aligned}
& \tilde{v}=\varepsilon \boldsymbol{U} \mathrm{e}^{\mathrm{i} t}+\mathrm{c.c} \cdot+\varepsilon^{2}\left(\boldsymbol{v}_{2}^{\mathrm{s}}+\mathrm{OT}\right)+\cdots, \\
& \tilde{w}=\varepsilon \dot{\mathrm{i}} F \mathrm{c}^{\mathrm{i} t}+\mathrm{c.c} .+\varepsilon^{2}(\boldsymbol{U} \cdot \tilde{\nabla} \bar{F}+\mathrm{c.c} .+\mathrm{OT})+\cdots, \\
& \partial \tilde{v} / \partial \xi=c \boldsymbol{H} \mathrm{c}^{\mathrm{i} t}+\text { c.c. }+\iota^{2}\left[\left(\partial \boldsymbol{v}_{3}^{\mathrm{s}} / \partial \eta\right)_{\eta=-\infty}+\mathrm{OT}\right]+\cdots,
\end{aligned}
$$

at $\xi=f$. Here $H$ is as defined in (5.14) and we have used the expression $\hat{\partial} \tilde{v} \hat{o} \xi=\mathscr{L} \tilde{v}+\tilde{\nabla} \tilde{w}+\mathrm{O}(|\xi|)$, which holds for the oscillatory part of the outer flow and is obtained when taking into account that this oscillatory flow is potential.

\subsection{Boundary conditions for the outer flow at the unperturbed boundary}

In order to calculate $\tilde{\boldsymbol{v}}, \tilde{w}$, and $\tilde{\tilde{v}} \tilde{\sigma} / \xi$ at the u.b. $(\xi=0)$ we need a leading order approximation of the derivatives of these quantities with respect to $\xi$, which are given by $(5.21)$ and

$$
\begin{aligned}
& \partial \tilde{w} / \partial \xi=-\hat{c}\left[(\tilde{\nabla} \cdot \mathbf{U}-2 \mathrm{i} M F) \mathrm{e}^{\mathrm{i} t}+\mathrm{c.c} .\right]+\cdots, \\
& \partial^{2} \tilde{\mathbf{v}} / \partial \xi^{2}=\varepsilon[2 \mathscr{L} \boldsymbol{H}-\tilde{\nabla}(\tilde{\nabla} \cdot \mathbf{U}-2 \mathrm{i} M F)] \mathrm{c}^{\mathrm{i} l}+\text { c.c. }+\cdots,
\end{aligned}
$$

at $\xi=f$, where we have used the continuity Eq. (3.16) and have taken into account (A.5) and (A.6) at leading order (recall that the oscillatory flow in the bulk is potential in first approximation). Then a Taylor expansion and (5.19)-(5.21) yield

$$
\begin{aligned}
& \tilde{\boldsymbol{v}}=\varepsilon \boldsymbol{U} \mathrm{c}^{\mathrm{i} t}+\mathrm{c.c} .+\varepsilon^{2}\left[\boldsymbol{y}_{2}^{\mathrm{s})}-(\bar{F} \boldsymbol{H}+\mathrm{c.c} .)+\mathrm{OT}\right]+\cdots, \\
& \tilde{w}=\imath \dot{\mathrm{i}} F \mathrm{c}^{\mathrm{i} t}+\text { c.c. }+\varepsilon^{2}[\tilde{\nabla} \cdot(\bar{F} \boldsymbol{U})+\text { c.c. }+\mathrm{OT}]+\cdots, \\
& \partial \tilde{v} / \partial \xi=\varepsilon \boldsymbol{H} \mathrm{e}^{\mathrm{i} t}+\text { c.c. }+\varepsilon^{2}\left(\hat{\partial} v_{3}^{\mathrm{s}} / \hat{\partial \eta}\right)_{\eta=-\infty} \\
& +c^{2}[\bar{F} \tilde{\nabla}(\tilde{\nabla} \cdot \mathbf{U})-2 \bar{F} \mathscr{L} \boldsymbol{H}-2 \mathrm{i} M \bar{F} \tilde{\nabla} F+\text { c.c. }+\mathrm{OT}]+\cdots,
\end{aligned}
$$

at the u.b. $(\xi=0)$, where we have used the identity $\tilde{\nabla}(\bar{F} \tilde{\nabla} \cdot \mathbf{U})=(\tilde{\nabla} \cdot \mathbf{U}) \tilde{\nabla} \bar{F}+\bar{F} \tilde{\nabla}(\tilde{\nabla} \cdot \mathbf{U})$. And we only need to apply matching conditions with the outer flow, invoking $(5.18),(5.23),(5.25)$ and 
(A.11), to obtain the right-hand sides in the boundary conditions $(3.14)$, which are

$$
\begin{aligned}
& \tilde{\tau}^{\mathrm{s} F}= \boldsymbol{\Phi} \equiv 2(2 M \bar{F}(\mathscr{L} \boldsymbol{U}+\mathrm{i} \tilde{\nabla} F)-\bar{F} \mathscr{L}(\mathscr{L} \boldsymbol{U})+\tilde{\nabla}[\tilde{\nabla} \cdot(\bar{F} \boldsymbol{U})]+(\tilde{\boldsymbol{\nabla}} \bar{F} \cdot \tilde{\nabla}) \boldsymbol{U}) \\
&+2((\tilde{\nabla} \cdot \mathrm{U}) \tilde{\nabla} \bar{F}-\mathrm{i}[(\mathscr{L} \boldsymbol{U} \cdot \dot{\nabla}) \overline{\boldsymbol{U}}+(\overline{\boldsymbol{U}} \cdot \tilde{\nabla}) \mathscr{L} \boldsymbol{U}+(\tilde{\boldsymbol{\nabla}} \cdot \overline{\boldsymbol{U}}) \mathscr{L} \boldsymbol{U}])+\mathrm{c.c}, \\
& \boldsymbol{v}^{\mathrm{s} \mathrm{E}} \cdot \mathbf{n}=\phi \equiv \tilde{\nabla} \cdot(\bar{F} \boldsymbol{U})+\mathrm{c.c},
\end{aligned}
$$

where we have taken into account that, since $\tilde{\nabla} \bar{F}$ and $\boldsymbol{U}$ are potential,

$$
\begin{gathered}
\bar{F} \tilde{\nabla} \cdot \mathbf{U}+\tilde{\nabla} \bar{F} \cdot \mathbf{U}=\tilde{\nabla} \cdot(\bar{F} \boldsymbol{U}),\left(\tilde{\nabla} \boldsymbol{U}+\tilde{\nabla} \boldsymbol{U}^{\top}\right) \cdot \tilde{\nabla} \bar{F}=2(\tilde{\nabla} \bar{F} \cdot \tilde{\nabla}) \boldsymbol{U}, \\
(\boldsymbol{U} \cdot \tilde{\nabla}) \tilde{\nabla} \bar{F}+(\tilde{\nabla} \bar{F} \cdot \tilde{\nabla}) \boldsymbol{U}=\tilde{\nabla}(\tilde{\nabla} \bar{F} \cdot \mathbf{U}) .
\end{gathered}
$$

Similarly, invoking (5.28b), (A.9) and (A.12), we obtain the counterparts of (5.26) and (5.27) for the Lagrangian velocity,

$$
\begin{aligned}
& \tilde{\tau}^{\mathrm{sL}}= 4(\bar{F}(2 M+\mathscr{L})(\mathscr{L} \boldsymbol{U}+\mathrm{i} \tilde{\nabla} F)+(\tilde{\nabla} \bar{F} \cdot \tilde{\nabla}) \boldsymbol{U} \\
&\left.+\left(\tilde{\nabla} \cdot \mathbf{U}^{\prime}\right) \tilde{\nabla} \bar{F}+\mathrm{i}[(\mathscr{L} \overline{\boldsymbol{U}} \cdot \tilde{\nabla}) \boldsymbol{U}-(\check{\nabla} \cdot \overline{\boldsymbol{U}}) \mathscr{L} \boldsymbol{U}]\right)+ \text { c.c. } \\
& \boldsymbol{v}^{\mathrm{sL}} \cdot \mathbf{n}=0 .
\end{aligned}
$$

Three remarks are now in order

(a) As in 2D (Longuet-Higgins, 1953), the Eulerian and Lagrangian shear stresses are independent of viscosity, but do depend explicitly on the curvature of the u.b., through the mean curvature $M$ and the Weingarten map $\mathscr{L}$.

(b) The normal component of the Lagrangian velocity vanishes at the u.b., which is consistent with the fact that the net mass flux across this surface must be zero. The Eulerian velocity instead does exhibit a normal component at the u.b. (see (5.27)), which is just due to non-linear terms in the boundary condition (3.3a) (or (3.20)); thus it comes from a purely inviscid effect, which is not affected by the oscillatory boundary laver.

(c) Let us assume that the oscillatory flow is standing, which occurs if the phases of $F$ and $\mathrm{i} U$ are constant and coincide. In this case, the right-hand sides of (5.26)-(5.27) and (5.29) identically vanish, which means that the normal component of the Eulerian velocity and the Eulcrian and Lagrangian shear stresses are all zero. This property is useful to obtain a priori propertics of the streaming flow produced by surface waves (Higuera et al, 2002a,b).

\section{3. $2 D$ problems, planes and cylinders}

The expression (5.30) simplifies in $2 \mathrm{D}$ to

$$
\tilde{\tau}^{\mathrm{sL}}=8[\kappa \bar{F}(\kappa U+\mathrm{i} \mathrm{d} F / \mathrm{d} s)+(\mathrm{d} \bar{F} / \mathrm{d} s+\mathrm{i} \kappa \bar{U}) \mathrm{d} U / \mathrm{d} s+\text { c.c. }],
$$

where we are using the same notation as at the end of Section 4 and $\kappa=\kappa \cdot n$ is the curvature of the u.b., with $\kappa$ and $n$ being the curvature vector and the outward unit normal, respectively; thus $\kappa>0$ if the u.b. is concave towards the exterior of the fluid domain. Here we have taken into account that in 2D the mean curvature $M$ must be replaced by $k / 2$ and that applying the Weingarten map consists of multiplying by $\kappa$. This expression coincides (up to notation differences) with that first 
derived by Longuet-Higgins (1953), which is usually employed in the literature (e.g. Phillips, 1977; Craik, 1982, 1985). The counterpart of (5.31) for the Eulcrian mean velocity is obtained from $(5.26)$ to be

$$
\tilde{\tau}^{\mathrm{sF}}=2\left[\mathrm{i} \kappa \bar{F} \mathrm{~d} F / \mathrm{d} s+\mathrm{d}^{2}(\bar{F} U) / \mathrm{d} s^{2}+(2 \mathrm{~d} \bar{F} / \mathrm{d} s+\mathrm{i} \kappa \bar{U}) \mathrm{d} U / \mathrm{d} s+\mathrm{c} . \mathrm{c} .\right] .
$$

If (in 3D) the u.b. is a plane, both the mean curvature and the Weingarten map identically vanish, and (5.26) and (5.29) simplify to

$$
\begin{aligned}
& \tilde{\tau}^{\mathrm{s}}=2[\tilde{\nabla}(\tilde{\nabla} \cdot(\bar{F} \boldsymbol{U}))+(\tilde{\nabla} \bar{F} \cdot \tilde{\nabla}) \boldsymbol{U}+(\tilde{\nabla} \cdot \mathbf{U}) \tilde{\nabla} \bar{F}+\text { c.c. }], \\
& \tilde{\tau}^{\mathrm{sL}}=4[(\tilde{\nabla} \bar{F} \cdot \tilde{\nabla}) \boldsymbol{U}+(\tilde{\nabla} \cdot \mathbf{U}) \tilde{\nabla} \bar{F}+\text { c.c. }] .
\end{aligned}
$$

This latter expression coincides (up to notation differences) with that obtained by Liu (1977).

For cylinders of arbitrary cross-section, the components of the Eulerian tangential stress along the cross-section and the axis are

$$
\begin{aligned}
\tilde{\tau}_{\mathrm{cr} s s \mathrm{~s}}^{\mathrm{s}}= & 2 \mathrm{i} \kappa \bar{F} F_{\mathrm{s}}+2\left[(\bar{F} U)_{\mathrm{s}}+(\bar{F} V)_{y}\right]_{\mathrm{s}}+2\left(2 \bar{F}_{\mathrm{s}} U_{\mathrm{s}}+\bar{F}_{y} U_{y}+\bar{F}_{\mathrm{s}} V_{y}\right) \\
& -2 \mathrm{i} \kappa\left[\bar{V} U_{y}+U\left(\bar{U}_{\mathrm{s}}+\bar{V}_{y}\right)\right]+\text { c.c. }, \\
\tilde{\tau}_{\mathrm{axial}}^{\mathrm{sF}}= & 2 \mathrm{i} \kappa \bar{F} F_{y}+2\left[(\bar{F} U)_{\mathrm{s}}+(\bar{F} V)_{y}\right]_{y}+2\left(\bar{F}_{s} V_{\mathrm{s}}+2 \bar{F}_{y} V_{y}+\bar{F}_{y} U_{\mathrm{s}}\right) \\
& -2 \mathrm{i} \kappa U \bar{V}_{\mathrm{s}}+\text { c.c. },
\end{aligned}
$$

respectively, where $\kappa$ is the curvature of the cross-section (whose sign is defined as in (5.31)) and we have taken into account that the mean curvature of the cylinder is $M=\kappa / 2$, and according to (3.19) the Weingarten map is given by

$$
\left[\begin{array}{l}
(\mathscr{L} \boldsymbol{v})_{\text {cross }} \\
(\mathscr{L} v)_{\text {axial }}
\end{array}\right]=\left[\begin{array}{ll}
\kappa & 0 \\
0 & 0
\end{array}\right]\left[\begin{array}{l}
u \\
v
\end{array}\right] .
$$

Eq. (5.35) reduces to (5.32) if $V$ is set to zero and dependence on $y$ is eliminated. Note however that (5.35)-(5.36) are essentially different from the particularization of (5.33) to Cartesian coordinates, namely the former are not obtained from the latter by just replacing $x$ by s (as it happened with the tangential velocity in Section 4). This is so because (5.35)-(5.36) depend explicitly on the curvature of the cross-section, as anticipated above. For instance, if the cylinder is circular with radius 1 and the flow is axisymmetric, then the axial Eulerian stress at the u.b. is obtained from (5.36) by setting $\kappa=-1$ and $U=0$, and climinating dependence on $s$, as

$$
\tilde{\tau}_{\mathrm{axial}}^{\mathrm{sF}}=2\left[-\mathrm{i} \bar{F} F_{y}+(\bar{F} V)_{y y}+2 \bar{F}_{y} V_{y}+\text { c.c. }\right] \text {. }
$$

which does not coincide with its counterpart in 2D for straight u.b. (i.e., that obtained from $(5.32)$ when setting $\kappa=0$ and replacing $U$ and $s$ by $V$ and $y$, respectively). Eq. (5.38) coincides (up to notation differenees) with those derived by Nicolás and Vega (1996) for this particular geometry, but not with its counterpart used by Lyubimov et al. (1997), where Longuet-Higgins's 2D formula was (somewhat loosely) employed for the cylindrical geometry. For large frequency the oscillatory flow exhibits a short axial wavelength, much smaller than the transversal curvature radius of the eylinder. Then $y$-derivatives are large, the first term in the right-hand side of (5.38) can be neglected and both expressions coincide. 


\section{Deformable vibrating solid boundaries}

If a solid wall is not at rest then its motion must be taken into account. For completeness we also allow the wall to be deformable, and assume that it is vibrating in such a way that its deflection along the outer unit normal to its unperturbed position, $\boldsymbol{n}$, and its tangential velocity (i.e., perpendicular to n) are

$$
f=\varepsilon F \mathrm{e}^{\mathrm{i} t}+\text { c.c. }+\cdots, \quad \hat{\boldsymbol{v}}^{w}=\varepsilon \boldsymbol{U}^{w} \mathrm{e}^{\mathrm{i} t}+\text { c.c. }+\cdots .
$$

As in Sections 4 and 5 the tangential component of the outer flow at the unperturbed boundary is of the form $\tilde{v}=a\left(U \mathrm{e}^{\mathrm{i} t}+\mathrm{c.c}\right.$. $)+\cdots$. Proceeding as in Sections 4 and 5 , the right-hand sides of the boundary conditions $(3.13)$ at the u.b. are found to be ${ }^{1}$

$$
\begin{aligned}
\boldsymbol{v}^{\mathrm{s}} \cdot \mathbf{n} & =\phi \equiv \tilde{\nabla} \cdot(\bar{F} \boldsymbol{U})+\mathrm{c} . \mathrm{c} . \\
\tilde{\boldsymbol{v}}^{\mathrm{s}}= & \boldsymbol{\Phi} \equiv\left[4 M\left(\boldsymbol{U}-\boldsymbol{U}^{w}\right)-\mathscr{L} \boldsymbol{U}^{w}-\mathrm{i} \tilde{\nabla} F\right] \bar{F}-\left[\left(\overline{\boldsymbol{U}}-\overline{\boldsymbol{U}}^{w}\right) \cdot \tilde{\nabla}\right]\left(\boldsymbol{U}-\boldsymbol{U}^{w}\right) / 2 \\
& -\left[(2+3 \mathrm{i}) \tilde{\boldsymbol{V}} \cdot \overline{\boldsymbol{U}}-(2-\mathrm{i}) \tilde{\nabla} \cdot \overline{\boldsymbol{U}}^{w}\right]\left(\boldsymbol{U}-\boldsymbol{U}^{w}\right) / 2 \\
& +\mathrm{i}\left[(\overline{\boldsymbol{U}} \cdot \tilde{\nabla}) \boldsymbol{U}^{w}+\left(\boldsymbol{U}^{w^{w}} \cdot \tilde{\nabla}\right) \overline{\boldsymbol{U}}\right]+\mathrm{c} . \mathrm{c} .
\end{aligned}
$$

Here, as in Section $5, M$ and $\mathscr{L}$ are the mean curvature and the Weingarten map of the u.b., and $\tilde{\nabla}$. and $\tilde{\nabla}$ are the intrinsic divergence and gradient operators along the u.b.

Invoking (3.12), (6.2)-(6.3) and (A.9), the tangential and normal components of the Lagrangian mean velocitics are found to be

$$
\tilde{\boldsymbol{v}}^{\mathrm{s}}=\mathrm{i}(\overline{\boldsymbol{U}} \cdot \tilde{\nabla}) \boldsymbol{U}+\bar{F}(2 \mathscr{L} \boldsymbol{U}+\mathrm{i} \tilde{\nabla} F)+\mathrm{c.c} .+\tilde{\boldsymbol{v}}^{\mathrm{sF}} \quad \text { and } \quad \boldsymbol{v}^{\mathrm{sL}} \cdot \mathbf{n}=0,
$$

respectively, where $\tilde{\boldsymbol{v}}^{\mathrm{F}}$ is given by (6.3). As in Section 5.1 (and for the same reason), the Lagrangian mean normal velocity at the u.b. vanishes but the Eulerian mean normal velocity does not. The tangential components of both the Eulerian and Lagrangian mean velocities do depend explicitly on the curvature of the u.b., which is entirely due to the motion of the boundary (c.f. Section 4).

\section{1. $2 D$ problems, planes and cylinders}

In 2D, with the same notation as in Section 5.1, Eq. (6.3) reduces to

$$
\begin{aligned}
u^{\mathrm{s}}= & \kappa \bar{F}\left(2 U-3 U^{w}\right)-\mathrm{i} \bar{F} \mathrm{~d} F / \mathrm{d} s-3\left[\left(\bar{U}-\bar{U}^{w}\right) \mathrm{d}\left(U-U^{w}\right) / \mathrm{d} s\right] / 2 \\
& \left.-\mathrm{i}\left[\left(3 U-5 U^{w}\right) \mathrm{d} \bar{U} / \mathrm{d} s\right]-\left(3 U-U^{w}\right) \mathrm{d} \bar{U}^{w} / \mathrm{d} s\right] / 2+\text { c.c. }
\end{aligned}
$$

If (in 3D) the u.b. is a plane then Eq. (6.3) becomes

$$
\begin{aligned}
\hat{\boldsymbol{v}}^{\mathrm{E}}= & -\mathrm{i} \bar{F} \tilde{\nabla} F-\left[(2+3 \mathrm{i}) \tilde{\nabla} \cdot \overline{\boldsymbol{U}}-(2-\mathrm{i}) \tilde{\nabla} \cdot \overline{\boldsymbol{U}}^{w}\right]\left(\boldsymbol{U}-\boldsymbol{U}^{w}\right) / 2 \\
& -\left[\left(\overline{\boldsymbol{U}}-\overline{\boldsymbol{U}}^{w}\right) \cdot \tilde{\nabla}\right]\left(\boldsymbol{U}-\boldsymbol{U}^{w}\right) / 2+\mathrm{i}\left[(\overline{\boldsymbol{U}} \cdot \dot{\nabla}) \boldsymbol{U}^{w}+\left(\boldsymbol{U}^{w} \cdot \tilde{\nabla}\right) \overline{\boldsymbol{U}}\right]+\text { c.c. }
\end{aligned}
$$

\footnotetext{
${ }^{1}$ A detailed derivation can be obtained from the authors.
} 
Two particular cases are of practical interest. If the solid boundary exhibits no tangential velocity to leading order then $U^{w}=\mathbf{0}$, and

$$
\tilde{\boldsymbol{v}}^{\mathrm{s} \mathrm{E}}=-\mathrm{i} \bar{F} \tilde{\nabla} F-[(\overline{\boldsymbol{U}} \cdot \tilde{\nabla}) \boldsymbol{U}+(2+3 \mathrm{i})(\tilde{\nabla} \cdot \overline{\boldsymbol{U}}) \boldsymbol{U}] / 2+\text { c.c. }
$$

This expression coincides (up to notation differences) with that obtained for unperturbed plane walls by Lighthill (1992), who extended a former cxpression obtained by Hunt and Johns (1963) for the particular case $F=0$, and also with those obtained for circular cylinders, in the axisymmetric (Nicolás and Vega, 1996; Lyubimov et al., 1997) and non-axisymmetric (Higuera, 1998; Higuera et al., 2002a,b) cases. If instead the outer flow is at rest and the solid boundary oscillates only tangentially to itself, then $U=\mathbf{0}, F=0$, and

$$
\tilde{\boldsymbol{v}}^{\mathrm{FF}}=-\left[\left(\overline{\boldsymbol{U}}^{w} \cdot \tilde{\boldsymbol{\nabla}}\right) \boldsymbol{U}^{w}+(2-\mathrm{i})\left(\tilde{\nabla} \cdot \overline{\boldsymbol{U}}^{w}\right) \boldsymbol{U}^{w}+\mathrm{c.c} .\right] 2+\cdots
$$

This latter expression is of interest for instance in the analysis of the streaming flow produced in the surrounding air by Marangoni waves in not-too-thin soap films (Vega et al., 1998 and references therein ).

Finally, if the u.b. is a cylinder then the components of the Eulerian velocity along the cross-section and the axis of the cylinder are obtained from (6.3) without difficulty, but yield quite involved expressions. For simplicity we only consider the particular case in which the oscillatory axial velocities vary linearly with the axial coordinate (which require that in addition the tangential velocity components and the solid boundary deflection be independent of the axial coordinate), namely

$$
U=U(s), \quad U^{w}=U^{w}(s), \quad V=y V_{0}(s), \quad V^{w}=y V_{0}^{w}(s), \quad F=F(s),
$$

which is of interest in flows in blood vessels (Padmanabhan and Pedley, 1987). In this case (6.3) yields the following components of the Eulerian mean velocity

$$
\begin{aligned}
u^{\mathrm{sF}}= & {\left[\kappa\left(2 U-3 U^{w^{w}}\right)-\mathrm{i} \mathrm{d} F / \mathrm{d} s\right] \bar{F}-\left[\left(\bar{U}-\bar{U}^{w}\right) \mathrm{d}\left(U-U^{w}\right) / \mathrm{d} s\right] / 2 } \\
& -\left[(2+3 \mathrm{i})\left(\mathrm{d} \bar{U} / \mathrm{d} s+\bar{V}_{0}\right)-(2-\mathrm{i})\left(\mathrm{d} \bar{U}^{w} / \mathrm{d} s+\bar{V}_{0}^{w}\right)\right]\left(U-U^{w}\right) / 2 \\
& +\mathrm{i}\left(\bar{U} \mathrm{~d} U^{w} / \mathrm{d} s+U^{w} \mathrm{~d} \bar{U} / \mathrm{d} s\right)+\mathrm{c} . \mathrm{c} ., \\
v^{\mathrm{sF}}= & y\left(2 \kappa \bar{F}\left(V_{0}-V_{0}^{w}\right)-(2)^{-1}\left[\left(U-U^{w}\right) \mathrm{d}\left(\bar{V}_{0}-\bar{V}_{0}^{w}\right) / \mathrm{d} s+\left|V_{0}-V_{0}^{w^{\prime}}\right|^{2}\right]\right. \\
& -\left[(2+3 \mathrm{i})\left(\mathrm{d} \bar{U} / \mathrm{d} s+\bar{V}_{0}\right)-(2-\mathrm{i})\left(\mathrm{d} \bar{U}^{w} \mathrm{~d} s+\bar{V}_{0}^{w}\right)\right]\left(V_{0}-V_{0}^{w w}\right) / 2 \\
& \left.+\mathrm{i}\left(\bar{U} \mathrm{~d} V_{0}^{w} / \mathrm{d} s+U^{w} \mathrm{~d} \bar{V}_{0} / \mathrm{d} s+2 \bar{V}_{0} V_{0}^{w}\right)+\text { c.c. }\right),
\end{aligned}
$$

respectively. Note that $(6.10)$ coincides with (6.5) if $V_{0}$ and $V_{0}^{w}$ are set to zero. And $(6.10)-(6.11)$ coincide with the expressions used by Padmanabhan and Pedley (1987) if the oscillatory flow is standing (namely, if the phases of $F$ and $i \zeta$ are constant), which was the case in that work.

\section{Concluding remarks}

We have considered the streaming flow produced by a nearly inviscid oscillatory flow in general 3D geometries. We have gencralized the 2D well-known formulae (Schlichting, 1932; Longuet-Higgins, 1953 ) giving the complete set of boundary conditions (3.13)-(3.14), which are necessary to calculate 
the secondary streaming flow in the bulk (outside the oscillatory boundary layers), where it is governed by Eqs. (3.9) and (3.10). With these general formulae we can avoid the tedious and tricky boundary layer analysis that would be necessary in each particular $3 \mathrm{D}$ problem, in the same way as it happened with the former 2D formulac. This is specially useful in the weakly-non-linear analysis of nearly-inviscid vibrating systems with free boundaries, such as the Faraday system (Miles and Henderson, 1990), in which the streaming flow is not just a by-product, but can interact at leading order with the primary vibrating flow (Vega et al., 2001; Higuera et al., 2001; Martin et al., 2002; Knobloch and Vega, 2002; Knobloch et al., 2002). The derivation of the boundary conditions has required to calculate the streaming flow within the oscillatory boundary layers attached to 3D (deformable) solid walls and free boundaries, and apply matching conditions with the streaming flow in the bulk. As in 2D, the boundary conditions consist of imposing a tangential velocity and a shear stress near a no slip boundary and a free boundary, respectively, in terms of the solution of the linear problem giving the primary oscillatory flow. The only assumptions for the validity of the analysis above are those in (3.4). Thus the free boundary deflection can be large compared to the boundary layer thickness, which happens as the streaming flow Reynolds number is large (see (3.11)), as required in many applications. This generality required that the analysis of the inner structure of the boundary layer be made in an oscillating curvilinear coordinate system attached to the moving boundary.

The formulate derived in the paper have been written in terms of both the Eulerian and Lagrangian mean velocities, and have been compared to previous results in the literature for particular $2 \mathrm{D}$ and $3 \mathrm{D}$ geometries.

For simplicity we have considered a steady streaming flow. But this can also be slowly varying with time due to various reasons (instabilities of steady patterns, coupling to the primary oscillatory flow). The extension is straightforward. We only need to allow the streaming flow variables to also depend on the slow time variable

$$
\tau=\varepsilon^{2} t
$$

The resulting equations would only change in a term $\hat{\partial} \mathrm{v}^{\mathrm{s} E} / \partial \tau$ that must be added to the left-hand side of (3.10). The boundary conditions (3.13) and (3.14) instead remain unchanged.

\section{Acknowledgements}

We are indebted to professors Francisco Higuera and Edgar Knobloch for some useful comments on an earlier version of this paper.

\section{Appendix A.}

Here, we collect some vector identities used above, whose derivation is omitted because it requires an extensive use of non-intuitive analytical tools, which perhaps are not of much interest in themselves from a purely fluid dynamical point of view, ${ }^{2}$ As above, $\xi$ is a coordinate along the

\footnotetext{
${ }^{2}$ It can be obtained from the authors upon request.
} 
outward unit normal $n$ to the u.b. and the velocity vector in the original static reference frame is decomposed as

$$
\boldsymbol{v}=\tilde{\boldsymbol{v}}+\tilde{w} \boldsymbol{n},
$$

where $\tilde{w}=\boldsymbol{v} \cdot \boldsymbol{n}$ and $\tilde{\boldsymbol{v}}=\boldsymbol{v}-\tilde{w} \boldsymbol{n}$ are the velocity components along $\boldsymbol{n}$ and orthogonal to $\boldsymbol{n}$, respectively. The following identities hold

$$
\begin{aligned}
& \boldsymbol{\nabla} p=\tilde{\nabla} p+\xi \mathscr{L}(\tilde{\nabla} p)+(\partial p / \partial \xi) n+\mathrm{O}\left(|\xi|^{2}|p|\right) \\
& \boldsymbol{\nabla} \cdot \boldsymbol{v}=\tilde{\boldsymbol{\nabla}} \cdot \tilde{\boldsymbol{v}}+\partial \tilde{w} / \partial \xi-2 M \tilde{w}+O(|\xi|(|\tilde{\boldsymbol{v}}|+|\tilde{w}|)), \\
& (\boldsymbol{v} \cdot \nabla) \boldsymbol{v}=(\tilde{\boldsymbol{v}} \cdot \tilde{\nabla}) \tilde{\boldsymbol{v}}+\tilde{w}(\partial \tilde{\boldsymbol{v}} / \partial \xi-\mathscr{L} \tilde{\boldsymbol{v}}) \\
& +[\tilde{\boldsymbol{v}} \cdot(\tilde{\nabla} \tilde{w}+\mathscr{L} \tilde{\boldsymbol{v}})+\tilde{w} \partial \tilde{w} / \hat{\mathrm{C}}] \boldsymbol{n}+\mathrm{O}\left(|\xi|(|\tilde{\boldsymbol{v}}|+|\tilde{w}|)^{2}\right), \\
& \Delta \boldsymbol{v}=\hat{\jmath}^{2} \tilde{\boldsymbol{v}} / \partial \xi^{2}+\left(\hat{\jmath}^{2} \tilde{w} / \partial \xi^{2}\right) \boldsymbol{n}+\mathrm{O}(|\boldsymbol{v}|+|\tilde{w}|),
\end{aligned}
$$

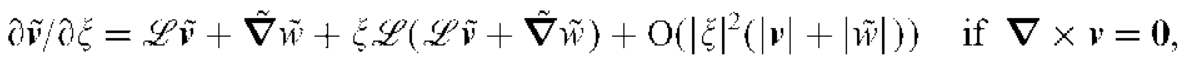

where $\tilde{\nabla}$. and $\tilde{\nabla}$ are the intrinsic divergence and gradient along the u.b. $\xi=0, M$ is the mean curvature of the u.b., $\mathscr{L}$ is the Weingarten map.

If $f$ is the (small) dellection of the free boundary along $\boldsymbol{n}$, then the boundary conditions (3.3a and b) can be written as

$$
\begin{aligned}
& \tilde{w}=\partial f / \partial t+\tilde{v} \cdot \tilde{\nabla} f, \\
& \partial \tilde{\boldsymbol{v}} / \partial \xi+\mathscr{L}(\tilde{\boldsymbol{v}}+f \mathscr{L} \tilde{\boldsymbol{v}})+\tilde{\nabla} \tilde{w}+f \mathscr{L}(\tilde{\nabla} \tilde{w})-\left(\tilde{\nabla} \tilde{\boldsymbol{v}}+\tilde{\nabla} \tilde{\boldsymbol{v}}^{\top}\right) \cdot \tilde{\nabla} f \\
& \quad+2(\partial \tilde{w} / \partial \xi) \tilde{\nabla} f+2 \tilde{w} \mathscr{L}(\tilde{\nabla} f)=O\left((|\tilde{\boldsymbol{v}}|+|\tilde{w}|)|f|^{2}\right) \text { at } \xi=f .
\end{aligned}
$$

The tangential and normal velocity components of the (re-scaled) Stokes drift are

$$
\tilde{\boldsymbol{v}}^{\mathrm{sd}}=\mathrm{i}(\overline{\boldsymbol{V}} \cdot \tilde{\nabla}) \boldsymbol{V}+\mathrm{i} \bar{W}(2 \mathscr{L} \boldsymbol{V}+\tilde{\nabla} W)+\mathrm{c} \cdot \mathrm{c} ., \quad \tilde{w}^{\mathrm{Sd}}=\mathrm{i} \tilde{\nabla} \cdot(W \overline{\boldsymbol{V}})+\mathrm{c} . \mathrm{c} .,
$$

in first approximation, in terms of the oscillatory flow velocity, assumed to be given by

$$
\tilde{v}=\varepsilon \boldsymbol{V} \mathrm{e}^{\mathrm{i} t}+\text { c.c. }+\cdots, \quad \tilde{w}=\varepsilon W \mathrm{e}^{\mathrm{i} t}+\text { c.c. }+\cdots .
$$

The tangential stress associated with the velocity field $v$ at the u.b. is

$$
\tilde{\tau}=\partial \tilde{v} / \partial \xi+\mathscr{L} \tilde{\boldsymbol{v}}+\tilde{\nabla} \tilde{w} .
$$

In particular, the tangential stress associated with the Stokes drift, namely the orthogonal projection of $\tau^{\mathrm{Sd}} \equiv\left[\nabla\left(\boldsymbol{v}^{\mathrm{Sd}}\right)+\nabla\left(v^{\mathrm{Sd}}\right)^{\top}\right] \cdot \mathbf{n}$ on the tangent plane to the u.b. is

$$
\begin{aligned}
\tilde{\tau}^{\mathrm{Sd}}= & 2([2(M+\mathscr{L})(\mathscr{L} \boldsymbol{U}+\mathrm{i} \tilde{\nabla} F)+\mathscr{L}(\mathscr{L} \boldsymbol{U})] \bar{F}-\tilde{\nabla}[\tilde{\nabla} \cdot(\bar{F} \boldsymbol{U})]+(\tilde{\nabla} \bar{F} \cdot \tilde{\nabla}) \boldsymbol{U} \\
& +(\tilde{\nabla} \cdot \mathbf{U}) \tilde{\nabla} \bar{F}+\mathrm{i}[(\mathscr{L} \overline{\boldsymbol{U}} \cdot \tilde{\nabla}) \boldsymbol{U}+(\overline{\boldsymbol{U}} \cdot \tilde{\nabla}) \mathscr{L} \boldsymbol{U}-(\tilde{\nabla} \cdot \overline{\boldsymbol{U}}) \mathscr{L} \boldsymbol{U}]+\text { c.c. })
\end{aligned}
$$


in first approximation, where with the notation used in this paper, $\boldsymbol{V}=\boldsymbol{U}$ and $W=\mathrm{i} F$, with $\boldsymbol{U}$ and $F$ given functions of position along the unperturbed free boundary.

\section{References}

Aris, R., 1962. Vectors, Tensors and the Basic Equations of Fluid Mechanics. Dover, New York.

Anilkumar, A.V., Grugel, R.N., Shen, X.F., Lee, C.P., Wang, T.G., 1993. Control of themocapillary convection in a liquid bridge by vibration. J. Appl. Phys. 73, 4165-4170.

Batchelor, G.K., 1967. An Introduction to Fluid Dynamics. Cambridge University Press, Cambridge.

Couder, Y., Chomaz, J.M., Rabaud, M., 1989. On the hydrodynamics of soap films. Physica D $37,384405$.

Craik, A.D.D., 1982. The drift velocity of water waves. I. Fluid Mech. 116, 187-205.

Craik, A.D.D., 1985. Wave Interactions and Fluid Flows. Cambridge University Press, Cambridge.

Faraday, M., 1831. On the forms and states assumed by fluids in contact with vibrating elastic surfaces. Philos. Trans. R. Soc. London $121,319-340$.

Glauert, M.B., 1957. A boundary layer theorem, with applications to rotating eylinders. J. Fluid Mech, 2, 89-99.

Higuera, M., 1998. Oscilaciones Débilmente no Lineales en Puentes Liquidos no Axilsimétricos. Doctoral Thesis, Universidad Politécnica de Madrid.

Higucra, M.J., Vega, J.M., Knobloch, E., 2001. Inleraction ol nearly-inviscid, multi-mode Faraday waves and mean flows. In: Bonilla, L.L., Platero, G., Reguera, D., Rubi, J.M. (Eds.), Coherent Structures in Complex Systems. Springer, Berlin, pp. $328 \quad 337$.

Higuera, M., Nicolás. J.A., Vega, J.M., 2002a. Weakly-non-linear non-axisymmetric oscillations of capillary bridges al small viscosity. Phys. Fluids 14, 3251-3271.

Higuera, M.J., Vega, J.M., Knobloch, E., 2002b. Coupled amplitude-mean flow equations for nearly-inviscid Faraday waves in moderate aspect ratio containers. J. Non-linear Sci. 12, 505-551.

Hunt, J.N., Johns, B., 1963. Currents induced by tides and gravily waves. Tellus 15, 343-351.

Iskandarani, M., Liu, P.L.F., 1991. Mass transport in three-dimensional water waves. J. Fluid Mech. $231,417437$.

Knobloch, I:, Vega, J.M., 2002. Nearly-inviscid Faraday waves. In: Newton, P., Ilolmes, P., Weinstein, A. (lids.), Goometry, Mechanics and Dynamies: Volume in Honor of the 60th Birthday of J.E. Marsden. Springer, Berlin, pp. 181222.

Knobloch, I:, Martel, C., Vega, I.M., 2002. Coupled amplitude-mean flow equations for nearly inviscid parametrically driven flows. Ammu. NY Acad. Sci, 974, 201-219.

Kuhlmann, H.C., 1999. Thermocapillary Convection in Models of Crystal Growth. Springer, Berlin.

Leibovich, S., 1983. On wave-current interaction theories of Langmuir circulations. Anul. Rev. Fluid Mech. 15, 391-427.

Lighthill, J., 1992. Acoustic streaming in the ear itsell. J. Fluid Mech. 239, 551-606.

Liu, P.L.-F., 1977. Mass transport in the free surface boundary layers. Coastal Eng. 17, 207219.

Liu, A.K., Davis, S.II., 1977. Viscous attenuation of mean drift in water waves. J. Fluid Mech. 81.63-84.

Longuet-Higgins, M.S., 1953. Mass transport in waler waves. Philos. Trans. R. Soc. A 245, 535-581.

Lord Rayleigh, J.W.S., 1883. The circulation of air observed in Kundt's tubes, and on some allied acoustical problems. Philos. Trans. R. Soc. London 175, 1-21.

Lyubimov, D., Lyubimova, T., Roux, B, 1997. Mechanisms of vibrational control of heat transler in a liquid bridge. Int. J. Heat Mass Transfer 40, 40314042.

Martin, E., Martel, C., Vega. J.M., 2002. Drift instability of standing Faraday waves in an annular container. I. Fluid Mech, 467, 57-79.

Mashayck, F., Ashgriz, N., 1998. Non-lincar oscillations of drops with intemal circulation. Phys, Fluids 10, $1071-1082$.

Miles, J., Henderson, D., 1990. Parametrically forced surface waves. Annu. Rev. Fluid Mech. $22,143165$.

Milewsky, P.A., Benney, D.I., 1995. Resonant interactions between vortical flows and water waves. Part I: deep water. Stud. Appl, Math, 94, 131-167.

Moffatt, H.H., 1964. Viscous and resistive eddies near a sharp corner. J. Fluid Mech. 18, l 18.

Mollot, D.I., Tsamopoulos, J., Chen, T.Y., Asligriz, A., 1993. Non-linear dynamics of capillary bridges: experiments. J. Fluid Mich, 255, 411-435 
Nicolas, J.A., Vege, J.M., 1996. Weakly non-linear oscillations of axisymmetric liquid bridges. J. Fluid Mech. 328 , 95128.

Nicolás, J.A., Rivas, D., Vega. J.M., 1997. The interaction of thermocapillary convection and low-frequency vibration in nearly-inviscid liquid bridges. \%. Angew. Math. Phys. 48, 389-423.

Nicolas, J.A., Rivas, D., Vega, J,M., 1998. On the steddy streaming flow due to high frequency vibration in nearly-inviscid liquid bridges. J. Fluid Mech. 354, 147-174.

Padmanabhan, N., Pedley, T.J., 1987. Three-dimensional steady streaming in a uniform tube with an oscillating elliptical cross-section. J. Fluid Mech, 178, 325-343.

Pedley, T.I., 2000. Blood flow in arteries and veins. In: Batchelor, G.K., Moffatt, II.K., Worster, M.G. (I:ds.), Perspectives in Fluid Dynamics: A Collective Introdnction to Current Research. Cambridge University Press, Cambridge, pp. $105-158$.

Phillips, O.M., 1977. The Dynamics of the Upper Ocem. Cambridge University Press, Cambridge.

Riley, N., 1992. Acoustic streaming about a cylinder in orthogonal beams. J. Fluid Mech. $242,387394$.

Riley, N., 2001. Steady streaming. Anmu. Rev. Fluid Mech. 33, 43-65.

Romano, A., 1993. Thermodynamics of Phase Transitions in Classical Field Theory. World Scientific, Singapore.

Schlichting, H, 1932. Berechnung ebener periodischer Grenzschichtströmungen. Phys. Z, 33, 327-335.

Schlichting, H., 1968. Boundary Layer Theory. McGraw-Hill, New York.

Smith, F.T., 1986. Steady and unsteady boundary-layer separation. Annu. Rev. Fluid Mech. 18, 197220

Sobey, I.J., 1980. On flow through furrowed channels. Part I. Calculated flow patterns. J. Fluid Mech. 96, 1-26.

Stephanoff, K.D., Sobey, I.J., Bellhouse, B.J., 1980. On flow through lumrowed channels. Part II. Observed llow palterns. I. Fluid Mich, 96, 27-32.

Stuart, J.T., 1966. Double boundary layers in oscillatory viscous flow. J. Fluid Mech. 24, 673687.

Thorpe, J.A., 1979. I'lementary Topics in Differential Geometry. Springer, Berlin.

Vega, J.M., Iliguera, F., Weidman, P.D., 1998. Quasi-steady vortical structures in vertically vibrating soap films. J. Fluid Mech. $372,213-230$.

Vega, J.M., Knobloch, E., Martel, C., 2001. Nearly inviscid Faraday waves in annular containers of moderately large aspect ratio. Physica D $154,313336$.

Yan, B., Ingham, D.B., Morton, B.R., 1993. Streaming flow induced by an oscillating cascade of circular cylinders. J. Fluid Mich, 252, 147-171 